\title{
Global classical solutions for partially dissipative hyperbolic system of balance laws
}

\author{
Jiang $\mathrm{Xu}^{*}$ \\ Department of Mathematics, \\ Nanjing University of Aeronautics and Astronautics, \\ Nanjing 211106, P.R.China \\ Shuichi Kawashima ${ }^{\dagger}$ \\ Graduate School of Mathematics, \\ Kyushu University, Fukuoka 812-8581, Japan
}

\begin{abstract}
This work is concerned with ( $N$-component) hyperbolic system of balance laws in arbitrary space dimensions. Under entropy dissipative assumption and the ShizutaKawashima algebraic condition, a general theory on the well-posedness of classical solutions in the framework of Chemin-Lerner's spaces with critical regularity is established. To do this, we first explore the functional space theory and develop an elementary fact that indicates the relation between homogeneous and inhomogeneous Chemin-Lerner's spaces. Then this fact allows to prove the local wellposedness for general data and global well-posedness for small data by using the Fourier frequency-localization argument. Finally, we apply the new existence theory to a specific fluid model-the compressible Euler equations with damping, and obtain the corresponding results in critical spaces.
\end{abstract}

Keywords: balance laws; entropy dissipative; classical solutions; Chemin-Lerner's spaces

AMS subject classification: 35L60, 35L45, 35F25

\footnotetext{
*E-mail: jiangxu_79@nuaa.edu.cn, jiangxu_79@yahoo.com.cn

${ }^{\dagger}$ E-mail: kawashim@math.kyushu-u.ac.jp
} 


\section{Introduction}

In this work, we consider the $N$-component hyperbolic system of balance laws, which are partial differential equations of the form

$$
U_{t}+\sum_{j=1}^{d} F^{j}(U)_{x_{j}}=G(U) .
$$

Here $U$ is the unknown $N$-vector valued function of time $t \geq 0$ and space coordinate $x=$ $\left(x_{1}, x_{2}, \cdots, x_{d}\right)(d \geq 1)$, taking values in an open convex set $\mathcal{O}_{U} \subset \mathbb{R}^{N}$ (the state space). $F^{j}$ and $G$ are given $N$-vector valued smooth functions on $\mathcal{O}_{U}$. The problem we are interested in is the Cauchy one of the system (1.1), so we supplement (1.1) with the following initial data

$$
U_{0}=U(0, x), \quad x \in \mathbb{R}^{d} .
$$

Note that in the absence of source term $G(U)$, (1.1) reduces to a system of conservation laws. In that case, it is well-known that classical solutions develop the singularity (e.g., shock wave) in finite time even when the initial data are small and smooth (see, e.g., [10]). System (1.1) with source terms typically govern non-equilibrium processes in physics for media with hyperbolic response as, for example, in gas dynamics. They also arise in the numerical simulation of conservation laws by relaxation schemes (see [1, 25, 37] and references cited therein). In these applications, the source term $G(U)$ has, or can be transformed by a linear transformation into, the form

$$
G(U)=\left(\begin{array}{c}
0 \\
g(U)
\end{array}\right)
$$

with $0 \in \mathbb{R}^{N_{1}}, g(U) \in \mathbb{R}^{N_{2}}$, where $N_{1}+N_{2}=N\left(N_{1} \neq 0\right)$. Obviously, the dissipation is not present in all the components of the system. A concrete example is the compressible Euler system with damping for perfect gas flow, see [32, 34] or Section 5] in this paper. As shown by [32, 34, the dissipative mechanisms due to the damping term, even if it enters only in the second equation, may prevent the formation of singularities and guarantee the global existence in time of classical solutions, at least for some restricted classes of initial data.

Inspired by the concrete example, a natural problem is that what conditions are posed on the general source term $G(U)$ such that it may prevent the finite time breakdown of classical solutions for the hyperbolic balance laws (1.1). A reasonable answer is that the system (1.1) has an entropy defined in [23] in a perfect manner and verifies the Shizuta-Kawashima ([SK]) stability condition formulated in 31 .

A notion of the entropy for (1.1) was first formulated by Chen, Levermore and Liu [8]. Their entropy was a natural extension of the classical one due to Godunov [15, Friedrichs and Lax [13] for hyperbolic conservation laws, i.e., (1.1) with $G(U) \equiv 0$, but it was not strong enough to develop the global existence theory for (1.1). Recently, under a technical requirement on the entropy dissipation and the [SK] stability condition, Yong in [36] proved the global existence of classical solutions in a neighborhood of a constant equilibrium $\bar{U} \in \mathbb{R}^{N}$ satisfying $G(\bar{U})=0$. Hanouzet and Natalini [17] obtained a similar existence result for one-dimensional problems in a similar situation. For the asymptotic behavior in time of the global solutions, in a similar situation, Bianchini, Hanouzet and Natalini [3] claimed the solutions approach the constant 
equilibrium state $\bar{U}$ in the $L^{p}$-norm at the rate $O\left(t^{-\frac{d}{2}\left(1-\frac{1}{p}\right)}\right)$, as $t \rightarrow \infty$, for $p \in[\min \{d, 2\}, \infty]$ by using the Duhamel principle and a detailed analysis of the Green kernel estimates for the linearized problem. Subsequently, the second author and Yong [24] removed the technical requirement on the entropy dissipation assumed in [36, 17, 3] by giving a perfect definition of the entropy for (1.1) and proved the same asymptotic decay estimate as in [3] under less regularity assumption on the initial data. The crucial point in 24] is to employ the time-weighted energy method which was first developed in [27] for compressible Navier-Stokes equations (see also [21]), and this enables us to show the decay estimate for $d \geq 2$ without assuming the $L^{1}$ property on the initial data.

It should be pointed out the above global existence and asymptotic behavior results of classical solutions were established in the framework of the existence theory of Kato and Majda [22, 26] for generally quasi-linear hyperbolic systems (i.e., $\mathcal{C}_{T} H^{s}\left(\mathbb{R}^{d}\right) \cap \mathcal{C}_{T}^{1} H^{s-1}\left(\mathbb{R}^{d}\right)$ ), where the regularity index $\sigma$ is required to be high $(s>1+d / 2)$. For the case of the critical regularity index $\sigma=1+d / 2$, are there the corresponding existence and stability for the balance laws (1.1)? To the best of our knowledge, this is a challenging open problem and few results are available in this direction. In the present paper, we shall explore the theory of functional spaces and try to solve the open problem with the aid of the notion of entropy, since it provides a proper setting to develop the existence theory for the balance laws (1.1) in [23, 36].

\subsection{Problem setting}

It is convenient to state basic ideas and main results of this paper, we first review the notion of entropy and the stability condition for (1.1) from [23, 24, 36]. To begin with, we set

$$
\mathcal{M}=\left\{\psi \in \mathbb{R}^{N}:\langle\psi, G(U)\rangle=0 \text { for any } U \in \mathcal{O}_{U}\right\},
$$

where the superscript ${ }^{\top}$ represents the transpose. Then $\mathcal{M}$ is a subset of $\mathbb{R}^{N}$ with $\operatorname{dim} \mathcal{M}=N_{1}$. In the discrete kinetic theory, $\mathcal{M}$ is called the space of summational (collision) invariants. From the definition of $\mathcal{M}$, we have

$$
G(U) \in \mathcal{M}^{\top} \text { (the orthogonal complement of } \mathcal{M} \text { ), for any } U \in \mathcal{O}_{U} \text {. }
$$

Moreover, corresponding to the orthogonal decomposition $\mathbb{R}^{N}=\mathcal{M} \oplus \mathcal{M}^{\top}$, we may write $U \in \mathbb{R}^{N}$ as

$$
U=\left(\begin{array}{c}
U_{1} \\
U_{2}
\end{array}\right)
$$

such that $U \in \mathcal{M}$ holds if and only if $U_{2}=0$. We denote by $\mathcal{E}$ the set of equilibrium state for the balance laws (1.1):

$$
\mathcal{E}=\left\{U \in \mathcal{O}_{U}: G(U)=0\right\} .
$$

In what follows, we give the notion of entropy.

Definition 1.1. ([23]) Let $\eta=\eta(U)$ be a smooth function defined in a convex open set $\mathcal{O}_{U} \subset$ $\mathbb{R}^{N}$. Then $\eta=\eta(U)$ is called an entropy for the balance laws (1.1) if the following statements hold: 
$(\bullet) \eta=\eta(U)$ is strictly convex in $\mathcal{O}_{U}$ in the sense that the Hessian $D_{U}^{2} \eta(U)$ is positive definite for $U \in \mathcal{O}_{U}$;

$(\bullet) D_{U} F_{j}(U)\left(D_{U}^{2} \eta(U)\right)^{-1}$ is symmetric for $U \in \mathcal{O}_{U}$ and $j=1, \ldots, d$;

$(\bullet) U \in \mathcal{E}$ if and only if $\left(D_{U} \eta(U)\right)^{\top} \in \mathcal{M}$;

$(\bullet)$ For $U \in \mathcal{E}$, the matrix $D_{U} G(U)\left(D_{U}^{2} \eta(U)\right)^{-1}$ is symmetric and nonpositive definite, and its null space coincides with $\mathcal{M}$.

Here and below, $D_{U}$ stands for the (row) gradient operator with respect to $U$.

Remark 1.1. We would like to emphasize that Definition[1.1] is a perfect definition of the entropy for the balance laws (1.1), and was introduced in [23] as a modification of the one first formulated in [8]. Some different definitions of entropy were also introduced in the previous papers [36, 17, 3]. These definitions are, however, not good enough so that these papers have to assume additional entropy dissipative properties such as the property stated in Proposition 1.1 below to get their global existence and decay results. Note that we do not need to assume Proposition 1.1 for our purpose, since it directly follows from Definition 1.1 .

Let $\eta(U)$ be the above entropy defined and set

$$
W(U)=\left(D_{U} \eta(U)\right)^{\top} .
$$

It was shown in 23] that the mapping $W=W(U)$ is a diffeomorphism from $\mathcal{O}_{U}$ onto its range $\mathcal{O}_{W}$. Let $U=U(W)$ be the inverse mapping which is also a diffeomorphism from $\mathcal{O}_{W}$ onto its range $\mathcal{O}_{U}$. Then (1.1) can be rewritten as

$$
A^{0}(W) W_{t}+\sum_{j=1}^{d} A^{j}(W) W_{x_{j}}=H(W)
$$

with

$$
\begin{gathered}
A^{0}(W)=D_{W} U(W), \\
A^{j}(W)=D_{W} F^{j}(U(W))=D_{U} F^{j}(U(W)) D_{W} U(W), \\
H(W)=G(U(W)) .
\end{gathered}
$$

Moreover, let us define

$$
L(W):=-D_{W} H(W)=-D_{U} G(U(W)) D_{W} U(W) .
$$

By virtue of (1.3), we have $D_{W} U(W)=D_{U}^{2} \eta(U(W))^{-1}$. Then it is not difficult to see that (1.1) is a symmetric dissipative system in the sense defined as follows.

Definition 1.2. ([23]) The system [1.4] is called symmetric dissipative if the following statements hold:

$(\bullet) A^{0}(W)$ is symmetric and positive definite for $W \in \mathcal{O}_{W}$;

$(\bullet) A^{j}(W)$ is symmetric for $W \in \mathcal{O}_{W}$ and $j=1, \ldots, d$; 
$(\bullet) H(W)=0$ if and only if $W \in \mathcal{M}$;

$(\bullet)$ For $W \in \mathcal{M}$, the matrix $L(W)$ is symmetric and nonnegative definite, and its null space coincides with $\mathcal{M}$.

As shown by [23], the symmetrization of balances laws can be characterized by the existence of the entropy function.

Theorem 1.1. ([23]) The following two statements are equivalent:

(i) The System (1.1) has an entropy.

(ii) There is a diffeomorphism by which (1.1) is transformed to a symmetric dissipative system (1.4).

Also, we know from [23] that the source term $H(W)$ of the symmetric dissipative system (1.4) has a useful expression, which further leads to a qualitative estimate of the entropy production term $D_{U} \eta(U) G(U)=W^{\top} H(W)$. For clarity, we formulate them by a proposition.

Proposition 1.1. ([23]) Fixed $\bar{W} \in \mathcal{M}$. Then

$$
H(W)=-L W+r(W),
$$

where $L=L(\bar{W}), r(W) \in \mathcal{M}^{\top}$ for all $W \in \mathcal{O}_{W}$. Furthermore, it holds that

$$
|r(W)| \leq C|W-\bar{W}||(I-\mathcal{P}) W|
$$

and

$$
\langle W, H(W)\rangle \leq-C|(I-\mathcal{P}) W|^{2}
$$

for $W \in \mathcal{O}_{W}$ close to $\bar{W}$, where $I$ the identity mapping on $\mathbb{R}^{N}$ and $\mathcal{P}$ the orthogonal projection onto $\mathcal{M}$.

In order to obtain the effective a priori estimates to extend the local solutions, we also reduce (1.4) to a symmetric dissipative system of normal form in the sense defined below.

Definition 1.3. ([23]) The symmetric dissipative system (1.4) is said to be of the normal form if $A^{0}(W)$ is block-diagonal associated with orthogonal decomposition $\mathbb{R}^{N}=\mathcal{M} \oplus \mathcal{M}^{\perp}$.

Use the partition as

$$
U=\left(\begin{array}{c}
U_{1} \\
U_{2}
\end{array}\right), \quad W=\left(\begin{array}{l}
W_{1} \\
W_{2}
\end{array}\right)
$$

associated with orthogonal decomposition $\mathbb{R}^{N}=\mathcal{M} \oplus \mathcal{M}^{\perp}$. We consider the mapping $U \rightarrow V$ defined by

$$
V=\left(\begin{array}{c}
V_{1} \\
V_{2}
\end{array}\right)=\left(\begin{array}{c}
U_{1} \\
W_{2}
\end{array}\right)
$$

where $W_{2}=\left(D_{U_{2}} \eta(U)\right)^{\top}$. This is a diffeomorphism from $\mathcal{O}_{U}$ onto its range $\mathcal{O}_{V}$. Denote by $U=U(V)$ the inverse mapping which is a diffeomorphism from $\mathcal{O}_{V}$ onto its range $\mathcal{O}_{U}$. 
Hence, $W=W(V)$ is the diffeomorphism composed by $W=W(U)$ and $U=U(V)$. After straightforward calculations, we show that

$$
\tilde{A}^{0}(V) V_{t}+\sum_{j=1}^{d} \tilde{A}^{j}(V) V_{x_{j}}=\tilde{H}(V)
$$

with

$$
\begin{gathered}
\tilde{A}^{0}(V)=\left(D_{V} W\right)^{\top} A^{0}(W) D_{V} W, \\
\tilde{A}^{j}(V)=\left(D_{V} W\right)^{\top} A^{j}(W) D_{V} W, \\
\tilde{H}(V)=\left(D_{V} W\right)^{\top} H(W),
\end{gathered}
$$

where $W$ is evaluated at $W(V)$. Precisely,

Theorem 1.2. ([24]) The system (1.5) is the symmetric dissipative system of the normal form and $\tilde{H}(V)=H(W)$. It holds $W \in \mathcal{M}$ if and only if $V \in \mathcal{M}$ between the variables $W$ with $V$. Furthermore, the matrix $\tilde{L}(V):=-D_{V} \tilde{H}(V)$ can be expressed as

$$
\tilde{L}(V)=\left(D_{V} W\right)^{\top} L(W) D_{V} W
$$

and satisfies $\tilde{L}(V)=L(W)$ if $V \in \mathcal{M}$ (i.e., $W \in \mathcal{M})$.

As a direct consequence, we have an analogue of Proposition 1.1

Corollary 1.1. Fixed $\bar{V} \in \mathcal{M}$. Then

$$
\tilde{H}(V)=-L V+\tilde{r}(V)
$$

where $L=L(\bar{W}), \tilde{r}(V) \in \mathcal{M}^{\top}$ for all $V \in \mathcal{O}_{V}$. Furthermore,

$$
|\tilde{r}(V)| \leq C|V-\bar{V}||(I-\mathcal{P}) V|
$$

and

$$
\langle V, \tilde{H}(V)\rangle \leq-C|(I-\mathcal{P}) V|^{2}
$$

for $V \in \mathcal{O}_{V}$ close to $\bar{V}$, where $I$ the identity mapping on $\mathbb{R}^{N}$ and $\mathcal{P}$ the orthogonal projection onto $\mathcal{M}$.

Finally, we formulate the [SK] stability condition for (1.5), since we deal with the symmetric dissipative system of normal form in the subsequent analysis. Let $\bar{V} \in \mathcal{M}$ be a constant state and consider the linearized form of (1.5) at $V=\bar{V}$ :

$$
\tilde{A}^{0} V_{t}+\sum_{j=1}^{d} \tilde{A}^{j} V_{x_{j}}+L V=0,
$$

where $\tilde{A}^{0}=\tilde{A}^{0}(\bar{V}), \tilde{A}^{j}=\tilde{A}^{j}(\bar{V})$ and $L=L(\bar{W})$. Taking the Fourier transform on (1.6) with respect to $x \in \mathbb{R}^{d}$, we obtain

$$
\tilde{A}^{0} \hat{V}_{t}+i|\xi| A(\omega) \hat{V}+L \hat{V}=0
$$


where $\tilde{A}(\omega):=\sum_{j=1}^{d} \tilde{A}^{j} \omega_{j}$ with $\omega=\xi /|\xi| \in \mathbb{S}^{d-1}$ (the unit sphere in $\mathbb{R}^{d}$ ). Let $\lambda=\lambda(i \xi)$ be the eigenvalues of (1.7), which solves the characteristic equation

$$
\operatorname{det}\left(\lambda \tilde{A}^{0}+i|\xi| \tilde{A}(\omega)+L\right)=0 .
$$

Then the stability condition for (1.6) is stated as follows.

Definition 1.4. The symmetric form (1.5) satisfies the stability condition at $\bar{V} \in \mathcal{M}$ if the following holds true: Let $\phi \in \mathbb{R}^{N}$ satisfies $\phi \in \mathcal{M}$ (i.e., $L \phi=0$ ) and $\lambda \tilde{A}^{0}+\tilde{A}(\omega) \phi=0$ for some $(\lambda, \omega) \in \mathbb{R} \times \mathbb{S}^{d-1}$, then $\phi=0$.

The stability condition was first formulated in [31] for symmetric hyperbolic-parabolic coupled systems including our present symmetric hyperbolic system (1.4) or (1.5). In addition, the characterization of the stability condition was also given by [31].

Theorem 1.3. The following statements are equivalent:

(•) The system (1.5) satisfies the stability condition at $\bar{V} \in \mathcal{M}$;

$(\bullet) \operatorname{Re} \lambda(i \xi)<0$ for $\xi \neq 0$;

(•) There is a positive constant c such that $\operatorname{Re} \lambda(i \xi) \leq-c|\xi|^{2} /\left(1+|\xi|^{2}\right)$ for $\xi \in \mathbb{R}^{d}$;

(•) There is an $N \times N$ matrix $\tilde{K}(\omega)$ depending smooth on $\omega \in \mathbb{S}^{d-1}$ satisfying the properties: (i) $\tilde{K}(-\omega)=-\tilde{K}(\omega)$ for $\omega \in \mathbb{S}^{d-1}$;

(ii) $\tilde{K}(\omega) \tilde{A}^{0}$ is skew-symmetric for $\omega \in \mathbb{S}^{d-1}$;

(iii) $[\tilde{K}(\omega) A(\omega)]^{\prime}+L$ is positive definite for $\omega \in \mathbb{S}^{d-1}$, where $[X]^{\prime}$ denotes the symmetric

part of the matrix $X$.

Remark 1.2. We also formulate the stability condition for (1.4) at the constant state $\bar{W} \in \mathcal{M}$. It turns out that the stability condition for (1.4) at the constant state $\bar{W} \in \mathcal{M}$ is equivalent to the stability condition for (1.5) at $\bar{V} \in \mathcal{M}$.

\subsection{Main results}

Recently, there are many well-posedness studies on the extension of the regularity class of initial data, such as using Besov spaces, or Triebel-Lizorkin spaces (see, e.g. [5, 6, 11, 35] and references therein). Most of those results are concerned on specific equations. In this paper, we confine the attention to a rather general type of equations as (1.1)-(1.2), furthermore, we study the case of critical regularity index $(\sigma=1+d / 2)$ where the classical existence theory of Kato and Majda fails. In this direction, there are only partial results available. In [20], Iftimie first considered (1.5) with $\tilde{A}^{0}(V)=I_{N}$ and gave a local existence for symmetric conservation laws pertaining to data in the Besov space $B_{2,1}^{1+d / 2}\left(\mathbb{R}^{d}\right)$, which is a subalgebra embedded in $\mathcal{C}^{1}\left(\mathbb{R}^{d}\right)$, and the lower bound of the maximal time of existence was also obtained. Using the standard iterative method, Chae 4 ] established a similar local existence for (1.5) independently, where he assumed that the condition $C^{-1} I_{N} \leq \tilde{A}^{0}(V) \leq C I_{N}\left(\forall V \in \mathbb{R}^{N}\right)$. In their works, they both considered the symmetric conservation laws (1.5), i.e. without the source term $\tilde{H}(V)$. However, up to now, the well-posedness and stability theory for general balance laws in critical spaces still are unknown. 
The balance laws (1.1) with an entropy can be symmetrized, however, the local existence of classical solutions of this paper does not follow from the works of Iftimie and Chae [20, 4] directly. Actually, we can remove their crucial assumption $C^{-1} I_{N} \leq \tilde{A}^{0}(V) \leq C I_{N}$, although it is satisfied by many concrete examples. We use the classical iteration argument and Friedrichs' regularization method to obtain the local existence. To develop the global local existence of classical solutions in critical spaces, the main ingredient is to construct uniform a priori estimates independent of time $T$ according to the dissipative mechanisms produced by source terms. Due to the partially dissipative structure of source term $Q(U)$, there occurs a technical obstruction. Precisely, we only capture the dissipation rate from the partial components $(I-\mathcal{P}) U$ rather than the total solutions $U$, which leads to the absence of the low-frequency part $\Delta_{-1}(\mathcal{P} U)$ in frequency-localization estimates. It seems that there is no chance to obtain a priori estimates from the standard definition of the critical Besov space $B_{2,1}^{1+d / 2}\left(\mathbb{R}^{d}\right)$. Fortunately, the time-space Besov spaces (Chemin-Lerner's spaces) $\widetilde{L}_{T}^{\rho}\left(B_{p, r}^{s}\right)$ help us to overcome the difficulty. The Chemin-Lerner's spaces were first introduced in [5] by Chemin and Lerner, which is the refinement of the usual spaces $L_{T}^{\rho}\left(B_{p, r}^{s}\right)$. Furthermore, we explore the functional space theory and develop a basic fact that indicates the relation between homogeneous and inhomogeneous Chemin-Lerner's spaces, see Proposition 6.1 in Appendix. Then it follows from this fact that some frequency-localization estimates in Chemin-Lerner's spaces with critical regularity are established effectively.

Our main results are stated as follows, where the regularity index $\sigma=1+d / 2$. First of all, we state the local well-posedness theorem of classical solutions to the Cauchy problem (1.1)-(1.2).

Theorem 1.4. Suppose the balance laws (1.1) admits an entropy defined by Definition 1.1. Let $\bar{U} \in \mathcal{E}$ be a constant state. If the initial date $U_{0}$ satisfy $U_{0}-\bar{U} \in B_{2,1}^{\sigma}\left(\mathbb{R}^{d}\right)$ and take values in a compact subset of $\mathcal{O}_{U}$, then there exists a time $T_{1}>0$ such that

(i) Existence: the Cauchy problem (1.1)-(1.2) has a unique solution $U \in \mathcal{C}^{1}\left(\left[0, T_{1}\right] \times \mathbb{R}^{d}\right)$ belongs to

$$
U-\bar{U} \in \widetilde{\mathcal{C}}_{T_{1}}\left(B_{2,1}^{\sigma}\left(\mathbb{R}^{d}\right)\right) \cap \widetilde{\mathcal{C}}_{T_{1}}^{1}\left(B_{2,1}^{\sigma-1}\left(\mathbb{R}^{d}\right)\right) .
$$

(ii) Blow-up criterion: there exists a constant $C_{0}>0$ such that the maximal time $T^{*}$ of existence of such a solution can be bounded from below by $T^{*} \geq \frac{C_{0}}{\left\|U_{0}-U\right\|_{B_{2,1}^{\sigma}}}$. Moreover, if $T^{*}$ is finite, then

$$
\limsup _{t \rightarrow T^{*}}\|U-\bar{U}\|_{B_{2,1}^{\sigma}}=\infty
$$

if and only if

$$
\int_{0}^{T^{*}}\|\nabla U\|_{L^{\infty}} d t=\infty
$$

Remark 1.3. The local existence result of classical solutions holds true in the framework of Chemin-Lerner's space with critical regularity, which can be proved by the classical iteration argument with the help of entropy notion, see Proposition 3.1 for details. Let us mention that the new general result can be regarded as an improvement of the works of Iftimie and Chae [20, 4], which enriches the classical local existence theory of Kato and Majda [22, 26].

In small amplitude regime, with the aid of the $[\mathrm{SK}]$ stability condition, we establish the global well-posedness of classical solutions to the Cauchy problem (1.1)-(1.2) in critical spaces. 
Theorem 1.5. Suppose the balance laws (1.1) admits an entropy defined as Definition 1.1 and the corresponding symmetric system (1.5) satisfies the stability condition at $\bar{V} \in \mathcal{M}$, where $\bar{V}$ is the constant state corresponding to $\bar{U}$. There exists a positive constant $\delta_{0}$ such that if

$$
\left\|U_{0}-\bar{U}\right\|_{B_{2,1}^{\sigma}\left(\mathbb{R}^{d}\right)} \leq \delta_{0}
$$

then the Cauchy problem (1.1)-(1.2) has a unique global solution $U \in \mathcal{C}^{1}\left(\mathbb{R}^{+} \times \mathbb{R}^{d}\right)$ satisfying

$$
U-\bar{U} \in \widetilde{\mathcal{C}}\left(B_{2,1}^{\sigma}\left(\mathbb{R}^{d}\right)\right) \cap \widetilde{\mathcal{C}}^{1}\left(B_{2,1}^{\sigma-1}\left(\mathbb{R}^{d}\right)\right) .
$$

Moreover, it holds that

$$
\begin{aligned}
& \|U-\bar{U}\|_{\widetilde{L}^{\infty}\left(B_{2,1}^{\sigma}\left(\mathbb{R}^{d}\right)\right)}+\mu_{0}\left(\|(I-\mathcal{P}) U\|_{\widetilde{L}^{2}\left(B_{2,1}^{\sigma}\left(\mathbb{R}^{d}\right)\right)}+\|\nabla U\|_{\widetilde{L}^{2}\left(B_{2,1}^{\sigma-1}\left(\mathbb{R}^{d}\right)\right)}\right) \\
\leq & C_{0}\left\|U_{0}-\bar{U}\right\|_{B_{2,1}^{\sigma}\left(\mathbb{R}^{d}\right)},
\end{aligned}
$$

where $C_{0}, \mu_{0}$ are some positive constants, and $\mathcal{P}$ is the orthogonal projection onto $\mathcal{M}$.

Remark 1.4. The proof of Theorem 1.5 relies on a crucial a priori estimate (Proposition 4.1) and the standard continuation argument. The a priori estimate can be done in three steps, which is derived by Fourier frequency-localization argument, rather than the classical energy approach as in [36]. The first step is the basic entropy variable estimate, which leads to the $L^{2}$-estimate exhibiting the dissipation rate of $(I-\mathcal{P}) U$. To take account of it, the next step is to estimate $(I-\mathcal{P}) U$ in homogeneous Chemin-Lerner's spaces with higher space derivatives. The last step is to capture the dissipation rate of $\nabla U$ in Fourier space, due to the important skew-symmetry condition in Theorem 1.3. To conclude, the a priori estimate is followed by Corollary 6.1,

Remark 1.5. In comparison with the previous efforts in [17, 23, 36], Theorem 1.5] exhibits the optimal critial regularity of the global existence of classical solutions, which can be regarded as a supplement to the existence theory of hyperbolic problems. On the other hand, we see that Theorem 1.5 is applicable to many concrete partially dissipative balance laws, for instance, the compressible Euler equation with damping in Sect. 5. However, let us mention that Theorem 1.5 was obtained by assuming all the time the [SK] condition. As a matter of fact, this condition is not satisfied by all physical models, such as the equations of gas dynamics in thermal nonequilibrium (see [37]). It would be interesting to weaken the condition while preserving the global existence in critical spaces. This issue is under current consideration.

As a direct consequence of Theorem 1.5, we can see the large-time asymptotic behavior of global solutions near the equilibrium $\bar{U}$ in some Besov spaces.

Corollary 1.2. Let $U$ be the solution in Theorem 1.5, Then

$$
\begin{gathered}
\|\nabla \mathcal{P} U(\cdot, t)\|_{B_{2,1}^{\sigma-1-\varepsilon}\left(\mathbb{R}^{d}\right)} \rightarrow 0, \\
\|\mathcal{P} U(\cdot, t)-\bar{U}\|_{B_{p, 2}^{\sigma-1-\varepsilon}\left(\mathbb{R}^{d}\right)} \rightarrow 0 \quad\left(p=\frac{2 d}{d-2}, d>2\right),
\end{gathered}
$$

and

$$
\|(I-\mathcal{P}) U(\cdot, t)\|_{B_{2,1}^{\sigma-\varepsilon}\left(\mathbb{R}^{d}\right)} \rightarrow 0
$$

for any $\varepsilon>0$, as $t \rightarrow+\infty$. 
The rest of this paper unfolds as follows. In Sect. 2, we introduce the Littlewood-Paley decomposition and recall the definitions and some useful conclusions in Besov spaces and CheminLerner's spaces. In Sect. 3, we give the local existence of classical solutions in Chemin-Lerner's spaces with critical regularity. Sect. 4 is devoted to the proof of a priori estimates. In Sect. 5, we present some applications about our new results. The paper ends with an Appendix (Sect. 6), where we develop the elementary fact that indicates the relation between homogeneous and inhomogeneous Chemin-Lerner's spaces, and establish the existence for linear symmetric system, which is used to prove the local existence for the quasilinear symmetric system (1.5).

Notations. Throughout the paper, we use $\langle\cdot, \cdot\rangle$ to denote the standard inner product in the real $\mathbb{R}^{N}$ or complex $\mathbb{C}^{N}$. C >0 stands for a generic constant, which might be different in each context. The notation $f \approx g$ means that $f \leq C g$ and $g \leq C f$. Denote by $\mathcal{C}([0, T], X)$ (resp., $\left.\mathcal{C}^{1}([0, T], X)\right)$ the space of continuous (resp., continuously differentiable) functions on $[0, T]$ with values in a Banach space $X$. For simplicity, the notation $\|(f, g)\|_{X}$ means $\|f\|_{X}+\|g\|_{X}$ with $f, g \in X$. In addition, we omit the space dependence, since all functional spaces (in $x$ ) are considered in $\mathbb{R}^{d}$.

\section{Littlewood-Paley theory and functional spaces}

The proofs of most of the results presented in this paper require a dyadic decomposition of Fourier variable, so we recall briefly the Littlewood-Paley decomposition theory and functional spaces, such as Besov spaces and Chemin-Lerner's spaces. The reader is also referred to [2] for details.

We start with the Fourier transform. The Fourier transform $\hat{f}$ of a $L^{1}$-function $f$ is given by

$$
\mathcal{F} f=\int_{\mathbb{R}^{d}} f(x) e^{-2 \pi x \cdot \xi} d x
$$

More generally, the Fourier transform of any $f \in \mathcal{S}^{\prime}$, the space of tempered distributions, is given by

$$
(\mathcal{F} f, g)=(f, \mathcal{F} g)
$$

for any $g \in \mathcal{S}$, the Schwartz class.

First, we fix some notation.

$$
\mathcal{S}_{0}=\left\{\phi \in \mathcal{S}, \partial^{\alpha} \mathcal{F} f(0)=0, \forall \alpha \in \mathbb{N}^{d} \text { multi-index }\right\} .
$$

Its dual is given by

$$
\mathcal{S}_{0}^{\prime}=\mathcal{S}^{\prime} / \mathbf{P}
$$

where $\mathbf{P}$ is the space of polynomials.

We now introduce a dyadic partition of $\mathbb{R}^{d}$. We choose $\phi_{0} \in \mathcal{S}$ such that $\phi_{0}$ is even,

$$
\operatorname{supp} \phi_{0}:=A_{0}=\left\{\xi \in \mathbb{R}^{d}: \frac{3}{4} \leq|\xi| \leq \frac{8}{3}\right\} \text {, and } \phi_{0}>0 \text { on } A_{0} \text {. }
$$

Set $A_{q}=2^{q} A_{0}$ for $q \in \mathbb{Z}$. Furthermore, we define

$$
\phi_{q}(\xi)=\phi_{0}\left(2^{-q} \xi\right)
$$


and define $\Phi_{q} \in \mathcal{S}$ by

$$
\mathcal{F} \Phi_{q}(\xi)=\frac{\phi_{q}(\xi)}{\sum_{q \in \mathbb{Z}} \phi_{q}(\xi)} .
$$

It follows that both $\mathcal{F} \Phi_{q}(\xi)$ and $\Phi_{q}$ are even and satisfy the following properties:

$$
\mathcal{F} \Phi_{q}(\xi)=\mathcal{F} \Phi_{0}\left(2^{-q} \xi\right), \quad \operatorname{supp} \mathcal{F} \Phi_{q}(\xi) \subset A_{q}, \quad \Phi_{q}(x)=2^{q d} \Phi_{0}\left(2^{q} x\right)
$$

and

$$
\sum_{q=-\infty}^{\infty} \mathcal{F} \Phi_{q}(\xi)=\left\{\begin{array}{lll}
1, & \text { if } & \xi \in \mathbb{R}^{d} \backslash\{0\} \\
0, & \text { if } \xi=0
\end{array}\right.
$$

As a consequence, for any $f \in S_{0}^{\prime}$, we have

$$
\sum_{q=-\infty}^{\infty} \Phi_{q} * f=f
$$

To define the homogeneous Besov spaces, we set

$$
\dot{\Delta}_{q} f=\Phi_{q} * f, \quad q=0, \pm 1, \pm 2, \ldots
$$

Definition 2.1. For $s \in \mathbb{R}$ and $1 \leq p, r \leq \infty$, the homogeneous Besov spaces $\dot{B}_{p, r}^{s}$ is defined by

$$
\dot{B}_{p, r}^{s}=\left\{f \in S_{0}^{\prime}:\|f\|_{\dot{B}_{p, r}^{s}}<\infty\right\},
$$

where

$$
\|f\|_{\dot{B}_{p, r}^{s}}=\left\{\begin{array}{l}
\left(\sum_{q \in \mathbb{Z}}\left(2^{q s}\left\|\dot{\Delta}_{q} f\right\|_{L^{p}}\right)^{r}\right)^{1 / r}, \quad r<\infty \\
\sup _{q \in \mathbb{Z}} 2^{q s}\left\|\dot{\Delta}_{q} f\right\|_{L^{p}}, \quad r=\infty
\end{array}\right.
$$

To define the inhomogeneous Besov spaces, we set $\Psi \in \mathcal{C}_{0}^{\infty}\left(\mathbb{R}^{d}\right)$ be even and satisfy

$$
\mathcal{F} \Psi(\xi)=1-\sum_{q=0}^{\infty} \mathcal{F} \Phi_{q}(\xi)
$$

It is clear that for any $f \in S_{0}^{\prime}$, yields

$$
\Psi * f+\sum_{q=0}^{\infty} \Phi_{q} * f=f .
$$

We further set

$$
\Delta_{q} f= \begin{cases}0, & j \leq-2, \\ \Psi * f, & j=-1, \\ \Phi_{q} * f, & j=0,1,2, \ldots\end{cases}
$$

Definition 2.2. For $s \in \mathbb{R}$ and $1 \leq p, r \leq \infty$, the inhomogeneous Besov spaces $B_{p, r}^{s}$ is defined by

$$
B_{p, r}^{s}=\left\{f \in S^{\prime}:\|f\|_{B_{p, r}^{s}}<\infty\right\}
$$

where

$$
\|f\|_{B_{p, r}^{s}}=\left\{\begin{array}{l}
\left(\sum_{q=-1}^{\infty}\left(2^{q s}\left\|\dot{\Delta}_{q} f\right\|_{L^{p}}\right)^{r}\right)^{1 / r}, \quad r<\infty \\
\sup _{q \geq-1} 2^{q s}\left\|\dot{\Delta}_{q} f\right\|_{L^{p}}, \quad r=\infty .
\end{array}\right.
$$


Let us point out that the definitions of $\dot{B}_{p, r}^{s}$ and $B_{p, r}^{s}$ does not depend on the choice of the Littlewood-Paley decomposition. Now, we state some basic conclusions, which will be used in subsequent analysis.

Lemma 2.1. (Bernstein inequality) Let $k \in \mathbb{N}$ and $0<R_{1}<R_{2}$. There exists a constant $C$, depending only on $R_{1}, R_{2}$ and $d$, such that for all $1 \leq a \leq b \leq \infty$ and $f \in L^{a}$,

$$
\operatorname{Supp} \mathcal{F} f \subset\left\{\xi \in \mathbb{R}^{d}:|\xi| \leq R_{1} \lambda\right\} \Rightarrow \sup _{|\alpha|=k}\left\|\partial^{\alpha} f\right\|_{L^{b}} \leq C^{k+1} \lambda^{k+d\left(\frac{1}{a}-\frac{1}{b}\right)}\|f\|_{L^{a}} ;
$$

$$
\operatorname{Supp} \mathcal{F} f \subset\left\{\xi \in \mathbb{R}^{d}: R_{1} \lambda \leq|\xi| \leq R_{2} \lambda\right\} \Rightarrow C^{-k-1} \lambda^{k}\|f\|_{L^{a}} \leq \sup _{|\alpha|=k}\left\|\partial^{\alpha} f\right\|_{L^{a}} \leq C^{k+1} \lambda^{k}\|f\|_{L^{a}}
$$

As a direct corollary of the above inequality, we have Remark 2.1. For all multi-index $\alpha$, it holds that

$$
\begin{gathered}
\frac{1}{C}\|f\|_{\dot{B}_{p, r}^{s+|\alpha|}} \leq\left\|\partial^{\alpha} f\right\|_{\dot{B}_{p, r}^{s}} \leq C\|f\|_{\dot{B}_{p, r}^{s+|\alpha|}} ; \\
\left\|\partial^{\alpha} f\right\|_{B_{p, r}^{s}} \leq C\|f\|_{B_{p, r}^{s+|\alpha|} .}
\end{gathered}
$$

The second one is the embedding properties in Besov spaces.

Lemma 2.2. Let $s \in \mathbb{R}$ and $1 \leq p, r \leq \infty$, then

(1) If $s>0$, then $B_{p, \tilde{r}}^{\tilde{s}}=L^{p} \cap B_{p, \tilde{r}}^{\tilde{s}}$;

(2) If $\tilde{s} \leq s$, then $B_{p, r}^{s} \hookrightarrow B_{p, \tilde{r}}^{\tilde{s}}$. This inclusion relation is false for the homogeneous Besov spaces;

(3) If $1 \leq r \leq \tilde{r} \leq \infty$, then $\dot{B}_{p, r}^{s} \hookrightarrow \dot{B}_{p, \tilde{r}}^{s}$ and $B_{p, r}^{s} \hookrightarrow B_{p, \tilde{r}}^{s}$;

(4) If $1 \leq p \leq \tilde{p} \leq \infty$, then $\dot{B}_{p, r}^{s} \hookrightarrow \dot{B}_{\tilde{p}, r}^{s-d\left(\frac{1}{p}-\frac{1}{\tilde{p}}\right)}$ and $B_{p, r}^{s} \hookrightarrow B_{\tilde{p}, r}^{s-d\left(\frac{1}{p}-\frac{1}{\tilde{p}}\right)}$;

(5) $\dot{B}_{p, 1}^{d / p} \hookrightarrow \mathcal{C}_{0}, \quad B_{p, 1}^{d / p} \hookrightarrow \mathcal{C}_{0}(1 \leq p<\infty) ;$

where $\mathcal{C}_{0}$ is the space of continuous bounded functions which decay at infinity.

The third one is the result of compactness in inhomogeneous Besov spaces.

Proposition 2.1. Let $1 \leq p, r \leq \infty, s \in \mathbb{R}$ and $\varepsilon>0$. For all $\phi \in C_{c}^{\infty}$, the map $f \mapsto \phi f$ is compact from $B_{p, r}^{s+\varepsilon}$ to $B_{p, r}^{s}$.

On the other hand, we also present the definition of Chemin-Lerner's space-time spaces first introduced by J.-Y. Chemin and N. Lerner [5], which are the refinement of the spaces $L_{T}^{\theta}\left(\dot{B}_{p, r}^{s}\right)$ or $L_{T}^{\theta}\left(B_{p, r}^{s}\right)$. 
Definition 2.3. For $T>0, s \in \mathbb{R}, 1 \leq r, \theta \leq \infty$, the homogeneous mixed time-space Besov spaces $\widetilde{L}_{T}^{\theta}\left(\dot{B}_{p, r}^{s}\right)$ is defined by

$$
\widetilde{L}_{T}^{\theta}\left(\dot{B}_{p, r}^{s}\right):=\left\{f \in L^{\theta}\left(0, T ; \mathcal{S}_{0}^{\prime}\right):\|f\|_{\widetilde{L}_{T}^{\theta}\left(\dot{B}_{p, r}^{s}\right)}<+\infty\right\}
$$

where

$$
\|f\|_{\widetilde{L}_{T}^{\theta}\left(\dot{B}_{p, r}^{s}\right)}:=\left(\sum_{q \in \mathbb{Z}}\left(2^{q s}\left\|\dot{\Delta}_{q} f\right\|_{L_{T}^{\theta}\left(L^{p}\right)}\right)^{r}\right)^{\frac{1}{r}}
$$

with the usual convention if $r=\infty$.

Definition 2.4. For $T>0, s \in \mathbb{R}, 1 \leq r, \theta \leq \infty$, the inhomogeneous mixed time-space Besov spaces $\widetilde{L}_{T}^{\theta}\left(B_{p, r}^{s}\right)$ is defined by

$$
\widetilde{L}_{T}^{\theta}\left(B_{p, r}^{s}\right):=\left\{f \in L^{\theta}\left(0, T ; \mathcal{S}^{\prime}\right):\|f\|_{\widetilde{L}_{T}^{\theta}\left(B_{p, r}^{s}\right)}<+\infty\right\}
$$

where

$$
\|f\|_{\widetilde{L}_{T}^{\theta}\left(B_{p, r}^{s}\right)}:=\left(\sum_{q \geq-1}\left(2^{q s}\left\|\Delta_{q} f\right\|_{L_{T}^{\theta}\left(L^{p}\right)}\right)^{r}\right)^{\frac{1}{r}}
$$

with the usual convention if $r=\infty$.

We further define

$$
\widetilde{\mathcal{C}}_{T}\left(B_{p, r}^{s}\right):=\widetilde{L}_{T}^{\infty}\left(B_{p, r}^{s}\right) \cap \mathcal{C}\left([0, T], B_{p, r}^{s}\right)
$$

and

$$
\widetilde{\mathcal{C}}_{T}^{1}\left(B_{p, r}^{s}\right):=\left\{f \in \mathcal{C}^{1}\left([0, T], B_{p, r}^{s}\right) \mid \partial_{t} f \in \widetilde{L}_{T}^{\infty}\left(B_{p, r}^{s}\right)\right\},
$$

where the index $T$ will be omitted when $T=+\infty$.

Next we state some basic properties on the inhomogeneous Chemin-Lerner's spaces only, since the similar ones follow in the homogeneous Chemin-Lerner's spaces.

The first one is that $\widetilde{L}_{T}^{\theta}\left(B_{p, r}^{s}\right)$ may be linked with the classical spaces $L_{T}^{\theta}\left(B_{p, r}^{s}\right)$ via the Minkowski's inequality:

Remark 2.2. It holds that

$$
\|f\|_{\widetilde{L}_{T}^{\theta}\left(B_{p, r}^{s}\right)} \leq\|f\|_{L_{T}^{\theta}\left(B_{p, r}^{s}\right)} \text { if } r \geq \theta ; \quad\|f\|_{\widetilde{L}_{T}^{\theta}\left(B_{p, r}^{s}\right)} \geq\|f\|_{L_{T}^{\theta}\left(B_{p, r}^{s}\right)} \text { if } r \leq \theta .
$$

Let us also recall the property of continuity for product in Chemin-Lerner's spaces $\widetilde{L}_{T}^{\theta}\left(B_{p, r}^{s}\right)$.

Proposition 2.2. The following inequality holds:

$$
\|f g\|_{\widetilde{L}_{T}^{\theta}\left(B_{p, r}^{s}\right)} \leq C\left(\|f\|_{L_{T}^{\theta_{1}}\left(L^{\infty}\right)}\|g\|_{\widetilde{L}_{T}^{\theta_{2}}\left(B_{p, r}^{s}\right)}+\|g\|_{L_{T}^{\theta_{3}\left(L^{\infty}\right)}}\|f\|_{\widetilde{L}_{T}^{\theta_{4}}\left(B_{p, r}^{s}\right)}\right)
$$

whenever $s>0,1 \leq p \leq \infty, 1 \leq \theta, \theta_{1}, \theta_{2}, \theta_{3}, \theta_{4} \leq \infty$ and

$$
\frac{1}{\theta}=\frac{1}{\theta_{1}}+\frac{1}{\theta_{2}}=\frac{1}{\theta_{3}}+\frac{1}{\theta_{4}}
$$

As a direct corollary, one has

$$
\|f g\|_{\widetilde{L}_{T}^{\theta}\left(B_{p, r}^{s}\right)} \leq C\|f\|_{\widetilde{L}_{T}^{\theta_{1}\left(B_{p, r}^{s}\right)}}\|g\|_{\widetilde{L}_{T}^{\theta_{2}}\left(B_{p, r}^{s}\right)}
$$

whenever $s \geq d / p, \frac{1}{\theta}=\frac{1}{\theta_{1}}+\frac{1}{\theta_{2}}$. 
Then we state a result of continuity for compositions in $\widetilde{L}_{T}^{\theta}\left(B_{p, r}^{s}\right)$.

Proposition 2.3. Let $s>0,1 \leq p, r, \theta \leq \infty, F \in W_{l o c}^{[s]+1, \infty}(I ; \mathbb{R})$ with $F(0)=0, T \in(0, \infty]$ and $v \in \widetilde{L}_{T}^{\theta}\left(B_{p, r}^{s}\right) \cap L_{T}^{\infty}\left(L^{\infty}\right)$. Then

$$
\|F(v)\|_{\widetilde{L}_{T}^{\theta}\left(B_{p, r}^{s}\right)} \leq C\left(1+\|v\|_{L_{T}^{\infty}\left(L^{\infty}\right)}\right)^{[s]+1}\|v\|_{\widetilde{L}_{T}^{\theta}\left(B_{p, r}^{s}\right)} .
$$

Finally, we present the estimates of commutators in Chemin-Lerner's spaces to end up this section. The indices $s, p$ behave just as in the stationary cases (see, e.g. 2, 11, 35]) whereas the time exponent $\theta$ behaves according to Hölder inequality.

Proposition 2.4. Let $1<p<\infty$ and $1 \leq \rho \leq \infty$. Then there exists a generic constant $C>0$ depending only on $s, d$ such that

$$
\left\{\begin{array}{l}
\left\|\left[f, \Delta_{q}\right] \mathcal{A} g\right\|_{L_{T}^{\theta}\left(L^{p}\right)} \leq C c_{q} 2^{-q s}\|\nabla f\|_{\widetilde{L}_{T}^{\theta_{1}}\left(B_{p, 1}^{s-1}\right)}\|g\|_{\widetilde{L}_{T}^{\theta_{2}\left(B_{p, 1}^{s}\right)}}, \quad s=1+\frac{d}{p}, \\
\left\|\left[f, \Delta_{q}\right] g\right\|_{L_{T}^{\theta}\left(L^{p}\right)} \leq C c_{q} 2^{-q(s+1)}\|f\|_{\widetilde{L}_{T}^{\theta_{1}\left(\dot{B}_{p, 1}^{\frac{d}{p}+1}\right)}}\|g\|_{\widetilde{L}_{T}^{\theta_{2}}\left(\dot{B}_{p, 1}^{s}\right)}, \quad s \in\left(-\frac{d}{p}-1, \frac{d}{p}\right],
\end{array}\right.
$$

where the commutator $[\cdot, \cdot]$ is defined by $[f, g]=f g-g f$, and the operator $\mathcal{A}:=\operatorname{div}$ or $\nabla .\left\{c_{q}\right\}$ denotes a sequence such that $\left\|\left(c_{q}\right)\right\|_{l^{1}} \leq 1, \frac{1}{\theta}=\frac{1}{\theta_{1}}+\frac{1}{\theta_{2}}$.

\section{Local existence}

In this section, we prove the local existence of classical solutions to the symmetric dissipative system (1.5) subject to the following initial data

$$
\left.V\right|_{t=0}=V_{0} \quad \text { with } \quad V_{0}=V\left(U_{0}\right),
$$

which can be regarded as an improvement of the works of Iftimie [20] and Chae [4] for symmetric hyperbolic systems. Here we could neglect the source term $\tilde{H}(V)$ in (1.5) for simplicity, since it is only responsible for the large-time behavior of solutions.

First, we consider the linear equations of (1.5):

$$
\tilde{A}^{0}(V) \hat{V}_{t}+\sum_{j=1}^{d} \tilde{A}^{j}(V) \hat{V}_{x_{j}}=0
$$

with

$$
\left.\hat{V}\right|_{t=0}=\hat{V}_{0}=V_{0}
$$

For the initial data $V_{0}$, we assume that $V_{0}-\bar{V} \in B_{2,1}^{\sigma}$ and

$$
V_{0}(x) \in \mathcal{O}_{0} \quad \text { for any } \quad x \in \mathbb{R}^{d}
$$

where $\mathcal{O}_{0}$ is a bounded open convex set in $\mathbb{R}^{N}$ satisfying $\overline{\mathcal{O}}_{0} \subset \mathcal{O}_{V}$.

For the existence of (3.2)-(3.3), the reader is referred to Proposition 6.2 in the Appendix. Furthermore, for $V(t, x)$, given function on $Q_{T}=[0, T] \times \mathbb{R}^{d}$, we assume that

$$
V-\bar{V} \in \widetilde{\mathcal{C}}_{T}\left(B_{2,1}^{\sigma}\right) \cap \widetilde{\mathcal{C}}_{T}^{1}\left(B_{2,1}^{\sigma-1}\right),
$$




$$
\begin{gathered}
V(t, x) \in \mathcal{O}_{1} \quad \text { for any }(t, x) \in Q_{T}, \\
\|V(t, x)-\bar{V}\|_{\widetilde{L}_{T}^{\infty}\left(B_{2,1}^{\sigma}\right)} \leq M_{1}, \\
\left\|\partial_{t} V(t, x)\right\|_{\widetilde{L}_{T}^{\infty}\left(B_{2,1}^{\sigma-1}\right)} \leq M_{2},
\end{gathered}
$$

where $\mathcal{O}_{1}$ is a bounded open convex set in $\mathbb{R}^{N}$ satisfying $\overline{\mathcal{O}}_{1} \subset \mathcal{O}_{V}$, and $M_{1}, M_{2}$ are two constants. Denote

$$
X_{T}^{\sigma}\left(\mathcal{O}_{1} ; M_{1}, M_{2}\right)=\left\{V \in \mathcal{O}_{V}: \text { the conditions (3.5) }-(3.8) \text { are satisfied }\right\} .
$$

Next, we shall prove that $X_{T}^{\sigma}\left(\mathcal{O}_{1} ; M_{1}, M_{2}\right)$ is an invariant set under iterations by determining $\mathcal{O}_{1}, M_{1}, M_{2}$ and $T$.

Lemma 3.1. (invariant set under iterations) Suppose that the initial data satisfy $V_{0}-\bar{V} \in B_{2,1}^{\sigma}$ and (3.4). Then there exists a time $T_{0}>0$, such that if $V \in X_{T_{0}}^{\sigma}\left(\mathcal{O}_{1} ; M_{1}, M_{2}\right)$, the Cauchy problem (3.2)-(3.3) has a unique solution $\hat{V}$ in the same $X_{T_{0}}^{\sigma}\left(\mathcal{O}_{1} ; M_{1}, M_{2}\right)$.

Proof. First, it follows from Proposition 6.2 and the assumption of Lemma 3.1 that $\hat{V}-\bar{V} \in$ $\widetilde{\mathcal{C}}_{T}\left(B_{2,1}^{\sigma}\right) \cap \widetilde{\mathcal{C}}_{T}^{1}\left(B_{2,1}^{\sigma-1}\right)$. Next, we show the solution $\hat{V}$ satisfies (3.7)-(3.8).

Set $\hat{Z}=\hat{V}-\bar{V}$. The system (3.2) can be written as

$$
\widetilde{A}^{0}(V) \hat{Z}_{t}+\sum_{j=1}^{d} \widetilde{A}^{j}(V) \hat{Z}_{x_{j}}=0
$$

Applying the operator $\Delta_{q}$ to (3.9), we infer that $\Delta_{q} \hat{Z}$ satisfies

$$
\widetilde{A}^{0}(V) \Delta_{q} \hat{Z}_{t}+\sum_{j=1}^{d} \widetilde{A}^{j}(V) \Delta_{q} \hat{Z}_{x_{j}}=-\sum_{j=1}^{d} \widetilde{A}^{0}(V)\left[\Delta_{q}, \widetilde{A}^{0}(V)^{-1} \widetilde{A}^{j}(V)\right] \hat{Z}_{x_{j}}
$$

where the commutator $[\cdot, \cdot]$ is defined by $[f, g]:=f g-g f$.

Perform the inter product with $\Delta_{q} \hat{Z}$ on both sides of the equation (3.10) to get

$$
\begin{aligned}
& \left\langle\widetilde{A}^{0}(V) \Delta_{q} \hat{Z}, \Delta_{q} \hat{Z}\right\rangle_{t}+\sum_{j=1}^{d}\left\langle\widetilde{A}^{j}(V) \Delta_{q} \hat{Z}, \Delta_{q} \hat{Z}\right\rangle_{x_{j}} \\
= & -2 \sum_{j=1}^{d}\left\langle\widetilde{A}^{0}(V)\left[\Delta_{q}, \widetilde{A}^{0}(V)^{-1} \widetilde{A}^{j}(V)\right] \hat{Z}_{x_{j}}, \Delta_{q} \hat{Z}\right\rangle+\left\langle\operatorname{div} \mathbb{A}(V) \Delta_{q} \hat{Z}, \Delta_{q} \hat{Z}\right\rangle,
\end{aligned}
$$

whereafter we use the notations:

$$
\mathbb{A}(V)=\left(\tilde{A}^{0}(V), \tilde{A}^{1}(V), \cdots, \tilde{A}^{d}(V)\right), \quad \operatorname{div} \mathbb{A}(V)=\tilde{A}^{0}(V)_{t}+\sum_{j=1}^{d} \tilde{A}^{j}(V)_{x_{j}}
$$


Integrating (3.11) over $\mathbb{R}^{d}$ gives

$$
\begin{aligned}
\frac{d}{d t}\left\|\Delta_{q} \hat{Z}\right\|_{L_{\widetilde{A}^{0}}^{2}}^{2}= & -2 \sum_{j=1}^{d} \int_{\mathbb{R}^{d}}\left\langle\widetilde{A}^{0}(V)\left[\Delta_{q}, \widetilde{A}^{0}(V)^{-1} \widetilde{A}^{j}(V)\right] \hat{Z}_{x_{j}}, \Delta_{q} \hat{Z}\right\rangle d x \\
& +\int_{\mathbb{R}^{d}}\left\langle\operatorname{div} \mathbb{A}(V) \Delta_{q} \hat{Z}, \Delta_{q} \hat{Z}\right\rangle d x
\end{aligned}
$$

with $\|f\|_{L_{\tilde{A}^{0}}^{2}}:=\left(\int_{\mathbb{R}^{d}}\left\langle\tilde{A}^{0}(V) f, f\right\rangle d x\right)^{1 / 2}$.

Since $V \in \mathcal{O}_{1}$ with $\overline{\mathcal{O}}_{1} \subset \mathcal{O}_{V}$, there exists a positive constant $C=C\left(\mathcal{O}_{1}, M_{1}\right)$ such that

$$
C^{-1} I_{N} \leq \tilde{A}^{0}(V) \leq C I_{N}
$$

which yields $\|f\|_{L_{\tilde{A}^{0}}^{2}} \approx\|f\|_{L^{2}}$.

Therefore, we have

$$
\begin{aligned}
\frac{d}{d t}\left\|\Delta_{q} \hat{Z}\right\|_{L^{2}}^{2}= & -2 \sum_{j=1}^{d} \int_{\mathbb{R}^{d}}\left\langle\widetilde{A}^{0}(V)\left[\Delta_{q}, \widetilde{A}^{0}(V)^{-1} \widetilde{A}^{j}(V)\right] \hat{Z}_{x_{j}}, \Delta_{q} \hat{Z}\right\rangle d x \\
& +\int_{\mathbb{R}^{d}}\left\langle\operatorname{div} \mathbb{A}(V) \Delta_{q} \hat{Z}, \Delta_{q} \hat{Z}\right\rangle d x \\
\leq & C\left\|\left[\Delta_{q}, \widetilde{A}^{0}(V)^{-1} \widetilde{A}^{j}(V)\right] \hat{Z}_{x_{j}}\right\|_{L^{2}}\left\|\Delta_{q} \hat{Z}\right\|_{L^{2}} \\
& +C\|\operatorname{div} \mathbb{A}(V)\|_{L^{\infty}}\left\|\Delta_{q} \hat{Z}\right\|_{L^{2}}^{2} .
\end{aligned}
$$

Let $\epsilon>0$ be a small number. Dividing (3.15) by $\left(\left\|\Delta_{q} \hat{Z}\right\|_{L^{2}}^{2}+\epsilon\right)^{1 / 2}$, we obtain

$$
\frac{d}{d t}\left(\left\|\Delta_{q} \hat{Z}\right\|_{L^{2}}^{2}+\epsilon\right)^{1 / 2} \leq C\left\|\left[\Delta_{q}, \widetilde{A}^{0}(V)^{-1} \widetilde{A}^{j}(V)\right] \hat{Z}_{x_{j}}\right\|_{L^{2}}+C\|\operatorname{div} \mathbb{A}(V)\|_{L^{\infty}}\left\|\Delta_{q} \hat{Z}\right\|_{L^{2}}
$$

A time integration yields

$$
\begin{aligned}
\left\|\Delta_{q} \hat{Z}\right\|_{L^{2}} \leq & \left(\left\|\Delta_{q} \hat{Z}\right\|_{L^{2}}^{2}+\epsilon\right)^{1 / 2} \\
\leq & \left(\left\|\Delta_{q} \hat{Z}_{0}\right\|_{L^{2}}^{2}+\epsilon\right)^{1 / 2}+C \int_{0}^{t}\left\|\left[\Delta_{q}, \widetilde{A}^{0}(V)^{-1} \widetilde{A}^{j}(V)\right] \hat{Z}_{x_{j}}\right\|_{L^{2}} d \tau \\
& +C \int_{0}^{t}\|\operatorname{div} \mathbb{A}(V)\|_{L^{\infty}}\left\|\Delta_{q} \hat{Z}\right\|_{L^{2}} d \tau .
\end{aligned}
$$

Taking the limit $\epsilon \rightarrow 0$ and using the estimates of commutators and continuity for the composition in the stationary case (see, e.g. [2, 35]), we arrive at

$$
\begin{aligned}
2^{q \sigma}\left\|\Delta_{q} \hat{Z}\right\|_{L_{t}^{\infty}\left(L^{2}\right)} \leq & 2^{q \sigma}\left\|\Delta_{q} \hat{Z}_{0}\right\|_{L^{2}}+C \int_{0}^{t} c_{q}(\tau)\|\nabla V\|_{B_{2,1}^{\sigma-1}}\|\hat{Z}\|_{B_{2,1}^{\sigma}} d \tau \\
& +C \int_{0}^{t} 2^{q \sigma}\|\operatorname{div} \mathbb{A}(V)\|_{L^{\infty}}\left\|\Delta_{q} \hat{Z}\right\|_{L^{2}} d \tau
\end{aligned}
$$


where we used Lemma 2.2 and $\left\|c_{q}(t)\right\|_{\ell^{1}} \leq 1$, for all $t \in[0, T]$. Summing up (3.17) on $q \geq-1$ implies

$$
\begin{aligned}
\|\hat{Z}\|_{\widetilde{L}_{T}^{\infty}\left(B_{2,1}^{\sigma}\right)} & \leq\left\|\hat{Z}_{0}\right\|_{B_{2,1}^{\sigma}}+C \int_{0}^{T}\left(\|\nabla V\|_{B_{2,1}^{\sigma}}+\|\operatorname{div} \mathbb{A}(V)\|_{L^{\infty}}\right)\|\hat{Z}(t)\|_{B_{2,1}^{\sigma}} d t \\
& \leq\left\|\hat{Z}_{0}\right\|_{B_{2,1}^{\sigma}}+C \int_{0}^{T}\left(\|\nabla V\|_{B_{2,1}^{\sigma}}+\|\operatorname{div} \mathbb{A}(V)\|_{L^{\infty}}\right)\|\hat{Z}\|_{\widetilde{L}_{t}^{\infty}\left(B_{2,1}^{\sigma}\right)} d t .
\end{aligned}
$$

Then it follows from Gronwall's inequality that

$$
\begin{aligned}
\|\hat{Z}\|_{\widetilde{L}_{T}^{\infty}\left(B_{2,1}^{\sigma}\right)} & \leq\left\|\hat{Z}_{0}\right\|_{B_{2,1}^{\sigma}} \exp \left\{C \int_{0}^{T}\left(\|\nabla V\|_{B_{2,1}^{\sigma}}+\|\operatorname{div} \mathbb{A}(V)\|_{L^{\infty}}\right) d t\right\} \\
& \leq e^{C T\left(M_{1}+M_{2}\right)}\left\|V_{0}-\bar{V}\right\|_{B_{2,1}^{\sigma}},
\end{aligned}
$$

since $V(x, t) \in X_{T}^{\sigma}\left(\mathcal{O}_{1} ; M_{1}, M_{2}\right)$. Take $T_{0}>0$ small such that

$$
e^{C T\left(M_{1}+M_{2}\right)} \leq 2
$$

Then the right side of (3.19) is bounded by $2\left\|V_{0}-\bar{V}\right\|_{B_{2,1}^{\sigma}}$, so we choose $M_{1}=2\left\|V_{0}-\bar{V}\right\|_{B_{2,1}^{\sigma}}$.

From the equations (3.2), we have

$$
\hat{V}_{t}+\sum_{j=1}^{d}\left\{\tilde{A}^{0}(V)^{-1} \tilde{A}^{j}(V)-\tilde{A}^{0}(\bar{V})^{-1} \tilde{A}^{j}(\bar{V})\right\} \hat{Z}_{x_{j}}=-\tilde{A}^{0}(\bar{V})^{-1} \tilde{A}^{j}(\bar{V}) \hat{Z}_{x_{j}}
$$

which implies from Proposition 2.2 that

$$
\begin{aligned}
\left\|\hat{V}_{t}\right\|_{\tilde{L}_{T_{0}}^{\infty}\left(B_{2,1}^{\sigma-1}\right)} & \leq C\left(\left\|\tilde{A}^{0}(V)^{-1} \tilde{A}^{j}(V)-\tilde{A}^{0}(\bar{V})^{-1} \tilde{A}^{j}(\bar{V})\right\|_{\widetilde{L}_{T_{0}}^{\infty}\left(B_{2,1}^{\sigma}\right)}+1\right)\left\|\hat{Z}_{x_{j}}\right\|_{\left.\widetilde{L}_{T_{0}\left(B_{2,1}^{\sigma}\right)}\right)} \\
& \leq C\left(\|V-\bar{V}\|_{\widetilde{L}_{T_{0}}^{\infty}\left(B_{2,1}^{\sigma}\right)}+1\right)\|V-\bar{V}\|_{\widetilde{L}_{T_{0}}^{\infty}\left(B_{2,1}^{\sigma}\right)} \\
& \leq C\left(1+M_{1}\right) M_{1}
\end{aligned}
$$

so we take $M_{2}=C\left(1+M_{1}\right) M_{1}$.

Finally, it can be shown that the solution $\hat{V}$ satisfies (3.6) . Indeed, from $\hat{V}_{t} \in \mathcal{C}\left(\left[0, T_{0}\right], B_{2,1}^{\sigma-1}\right)$ and the simply equality $\hat{V}-V_{0}=\int_{0}^{t} \hat{V}_{t} d \tau$, we deduce that

$$
\left|\hat{V}(t, x)-V_{0}(x)\right| \leq T_{0} \sup _{t \in\left[0, T_{0}\right]}\left\|\hat{V}_{t}(t, \cdot)\right\|_{L^{\infty}} \leq C T_{0} M_{2} .
$$

Note that (3.4), we take $T_{0}$ small so that $C T_{0} M_{2}<d_{1}$ with $d_{1}<d_{0}=\operatorname{dist}\left(\mathcal{O}_{0}, \partial \mathcal{O}_{V}\right)$, so $\hat{V} \in \mathcal{O}_{1}$ with $\mathcal{O}_{1}:=d_{1}$-neighborhood of $\mathcal{O}_{0}$. This completes the proof of Lemma 3.1

With the help of Lemma 3.1, we further establish the local existence of (1.5) with (3.1).

Proposition 3.1. Assume that the initial date $V_{0}$ satisfies $V_{0}-\bar{V} \in B_{2,1}^{\sigma}$ and 3.4). Then 
(i) Existence: there exists a positive constant $T_{1}\left(\leq T_{0}\right)$, depending only on $\mathcal{O}_{0}, d_{1}$ and $\| V_{0}-$ $\bar{V} \|_{B_{2,1}^{\sigma}}$ such that the system (1.5) with (3.1) has a unique solution $V \in X_{T_{1}}^{\sigma}\left(\mathcal{O}_{1} ; M_{1}, M_{2}\right)$, where $\mathcal{O}_{1}, M_{1}, M_{2}$ are determined by Lemma 3.1. In particular, the solution satisfies

$$
V \in \mathcal{C}^{1}\left(\left[0, T_{1}\right] \times \mathbb{R}^{d}\right)
$$

and

$$
V-\bar{V} \in \widetilde{\mathcal{C}}_{T_{1}}\left(B_{2,1}^{\sigma}\right) \cap \widetilde{\mathcal{C}}_{T_{1}}^{1}\left(B_{2,1}^{\sigma-1}\right) .
$$

(ii) Blow-up criterion: there exists a constant $C_{0}>0$ such that the maximal time $T^{*}$ of existence of such a solution can be bounded from below by $T^{*} \geq \frac{C_{0}}{\left\|V_{0}-V\right\|_{B_{2,1}^{\sigma}}}$. Moreover, if $T^{*}$ is finite, then

$$
\limsup _{t \rightarrow T^{*}}\|V-\bar{V}\|_{B_{2,1}^{\sigma}}=\infty
$$

if and only if

$$
\int_{0}^{T^{*}}\|\nabla V(t)\|_{L^{\infty}} d t=\infty .
$$

Proof. Based on Lemma 3.1, we introduce the successive approximation sequence $\left\{V^{n}(t, x)\right\}_{n=0}^{\infty}$ for the Cauchy problem (3.2)-(3.3) as follows:

$$
V^{0}=\bar{V},
$$

and for $n>0$,

$$
\tilde{A}^{0}\left(V^{n}\right) V_{t}^{n+1}+\sum_{j=1}^{d} \tilde{A}^{j}\left(V^{n}\right) V_{x_{j}}^{n+1}=0
$$

with the initial data

$$
\left.V^{n+1}\right|_{t=0}=V_{0}(x) \text {. }
$$

By Lemma 3.1, the sequence $\left\{V^{n}(t, x)\right\}_{n=0}^{\infty}$ is well defined on $Q_{T_{0}}$ for all $n \geq 0$, and is uniformly bounded with respect to $n \geq 0$, i.e., $V^{n} \in X_{T_{0}}^{\sigma}\left(\mathcal{O}_{1} ; M_{1}, M_{2}\right)$. Next, it will be shown that $\left\{V^{n}(t, x)\right\}_{n=0}^{\infty}$ is a Cauchy sequence in $\widetilde{\mathcal{C}}_{T_{0}}\left(B_{2,1}^{\sigma-1}\right)$. Define $\hat{V}^{n}=V^{n+1}-V^{n}$ for any $n \geq 1$.

Take the difference between the equation (3.23) for the $n+1$-th step and the $n$-th step to give

$$
\begin{aligned}
& \tilde{A}^{0}\left(V^{n}\right) \hat{V}_{t}^{n}+\sum_{j=1}^{d} \tilde{A}^{j}\left(V^{n}\right) \hat{V}_{x_{j}}^{n} \\
& =-\tilde{A}^{0}\left(V^{n}\right) \sum_{j=1}^{d}\left\{\tilde{A}^{0}\left(V^{n}\right)^{-1} \tilde{A}^{j}\left(V^{n}\right)-\tilde{A}^{0}\left(V^{n-1}\right)^{-1} \tilde{A}^{j}\left(V^{n-1}\right)\right\} V_{x_{j}}^{n}
\end{aligned}
$$

subject to the initial data

$$
\left.\hat{V}^{n}\right|_{t=0}=0
$$

Apply the operator $\Delta_{q}$ to (3.25) to get

$$
\tilde{A}^{0}\left(V^{n}\right) \Delta_{q} \hat{V}_{t}^{n}+\sum_{j=1}^{d} \tilde{A}^{j}\left(V^{n}\right) \Delta_{q} \hat{V}_{x_{j}}^{n}=R_{1}+R_{2},
$$


where

$$
\begin{gathered}
R_{1}:=-\sum_{j=1}^{d} \widetilde{A}^{0}\left(V^{n}\right)\left[\Delta_{q}, \widetilde{A}^{0}\left(V^{n}\right)^{-1} \widetilde{A}^{j}\left(V^{n}\right)\right] \hat{V}_{x_{j}}^{n}, \\
R_{2}:=-\sum_{j=1}^{d} \Delta_{q}\left\{\tilde{A}^{0}\left(V^{n}\right)\left(\tilde{A}^{0}\left(V^{n}\right)^{-1} \tilde{A}^{j}\left(V^{n}\right)-\tilde{A}^{0}\left(V^{n-1}\right)^{-1} \tilde{A}^{j}\left(V^{n-1}\right)\right) V_{x_{j}}^{n}\right\} .
\end{gathered}
$$

Following from the similar steps as (3.12)-(3.16), we get up with

$$
\left\|\Delta_{q} \hat{V}^{n}\right\|_{L^{2}} \leq C \int_{0}^{t}\left(\left\|R_{1}\right\|_{L^{2}}+\left\|R_{2}\right\|_{L^{2}}\right) d \tau+C \int_{0}^{t}\left\|\operatorname{div} \mathbb{A}\left(V^{n}\right)\right\|_{L^{\infty}}\left\|\Delta_{q} \hat{V}^{n}\right\|_{L^{2}} d \tau .
$$

By multiplying the factor $2^{q(\sigma-1)}$ on both sides of the inequality (3.28), we obtain

$$
\begin{aligned}
& 2^{q(\sigma-1)}\left\|\Delta_{q} \hat{V}^{n}\right\|_{L_{t}^{\infty}\left(L^{2}\right)} \\
\leq & C \int_{0}^{t} c_{q}\left\|\nabla V^{n}\right\|_{B_{2,1}^{\sigma-1}}\left\|\hat{V}^{n}\right\|_{B_{2,1}^{\sigma-1}} d \tau+C \int_{0}^{t} c_{q}\left\|\hat{V}^{n-1}\right\|_{B_{2,1}^{\sigma-1}}\left\|V^{n}-\bar{V}\right\|_{B_{2,1}^{\sigma}} d \tau \\
& +C \int_{0}^{t}\left\|\operatorname{div} \mathbb{A}\left(V^{n}\right)\right\|_{L^{\infty}} 2^{q(\sigma-1)}\left\|\Delta_{q} \hat{V}^{n}\right\|_{L^{2}} d \tau, \quad t \in\left[0, T_{0}\right],
\end{aligned}
$$

where $\left\{c_{q}\right\}$ denotes some sequence which satisfies $\left\|\left(c_{q}\right)\right\|_{\ell^{1}} \leq 1$.

Summing up (3.29) on $q \geq-1$, it is not difficult to get

$$
\begin{aligned}
\left\|\hat{V}^{n}\right\|_{\widetilde{L}_{T_{0}}^{\infty}\left(B_{2,1}^{\sigma-1}\right)} \leq & C \int_{0}^{T_{0}}\left(\left\|V^{n}-\bar{V}\right\|_{B_{2,1}^{\sigma}}+\left\|\operatorname{div} \mathbb{A}\left(V^{n}\right)\right\|_{L^{\infty}}\right)\left\|\hat{V}^{n}\right\|_{\widetilde{L}_{t}^{\infty}\left(B_{2,1}^{\sigma-1}\right)} d t \\
& +C \int_{0}^{T_{0}}\left\|V^{n}-\bar{V}\right\|_{B_{2,1}^{\sigma}}\left\|\hat{V}^{n-1}\right\|_{\widetilde{L}_{t}^{\infty}\left(B_{2,1}^{\sigma-1}\right)} d t .
\end{aligned}
$$

With the aid of Gronwall's inequality, we immediately deduce that

$$
\begin{aligned}
& \left\|\hat{V}^{n}\right\|_{\widetilde{L}_{T_{0}}^{\infty}\left(B_{2,1}^{\sigma-1}\right)} \\
\leq & C T_{0} e^{C \int_{0}^{T_{0}}\left(\left\|V^{n}-\bar{V}\right\|_{B_{2,1}^{\sigma}}+\left\|\operatorname{div} \mathbb{A}\left(V^{n}\right)\right\|_{\left.L^{\infty}\right)}\right) d t}\left\|V^{n}-\bar{V}\right\|_{\widetilde{L}_{T_{0}}^{\infty}\left(B_{2,1}^{\sigma}\right)}\left\|\hat{V}^{n-1}\right\|_{\widetilde{L}_{T_{0}}^{\infty}\left(B_{2,1}^{\sigma-1}\right)} \\
\leq & C M_{1} T_{0} e^{C T_{0}\left(M_{1}+M_{2}\right)}\left\|\hat{V}^{n-1}\right\|_{\widetilde{L}_{T_{0}}^{\infty}\left(B_{2,1}^{\sigma-1}\right)} .
\end{aligned}
$$

Take $T_{1}$ so small that

$$
T_{1} \leq T_{0} \text { and } C M_{1} T_{1} e^{C T_{1}\left(M_{1}+M_{2}\right)} \leq \frac{1}{2} .
$$

Then it follows from (3.31) that $\left(V^{n}-\bar{V}\right)$ is a Cauchy sequence in $\widetilde{\mathcal{C}}_{T_{1}}\left(B_{2,1}^{\sigma-1}\right)$. There exists a function $V(t, x)$ with $V-\bar{V} \in \widetilde{\mathcal{C}}_{T_{1}}\left(B_{2,1}^{\sigma-1}\right)$ such that $\left(V^{n}-V\right) \rightarrow 0$ strongly in $\widetilde{\mathcal{C}}_{T_{1}}\left(B_{2,1}^{\sigma-1}\right)$ as $n \rightarrow \infty$. On the other hand it follows from Fatou's property that the conditions (3.7)-(3.8) are satisfied, since $V^{n}$ is uniformly bounded in the space $X_{T_{0}}^{\sigma}\left(\mathcal{O}_{1} ; M_{1}, M_{2}\right) \subset X_{T_{1}}^{\sigma}\left(\mathcal{O}_{1} ; M_{1}, M_{2}\right)$. The property of strong convergence enable us to pass to the limits in the system (3.23)-(3.24) and conclude that $V$ is a solution to (1.5) and (3.1). What remains is to check that $V-\bar{V} \in$ $\mathcal{C}\left(\left[0, T_{1}\right], B_{2,1}^{\sigma}\right)$. Indeed, we easily achieve that the map $t \mapsto\left\|\Delta_{q}(V(t)-\bar{V})\right\|_{L^{2}}$ is continuous 
on $\left[0, T_{1}\right]$, since $V-\bar{V} \in \mathcal{C}\left(\left[0, T_{1}\right] ; B_{2,1}^{\sigma-1}\right)$. Then we have $\Delta_{q}(V(t)-\bar{V}) \in \mathcal{C}\left(\left[0, T_{1}\right] ; B_{2,1}^{\sigma}\right)$ for all $q \geq-1$. Note that $V-\bar{V} \in \widetilde{L}_{T_{1}}^{\infty}\left(B_{2,1}^{\sigma}\right)$, the series $\sum_{q \geq-1} 2^{q \sigma}\left\|\Delta_{q}(V(t)-\bar{V})\right\|_{L^{2}}$ converges uniformly on $\left[0, T_{1}\right]$, which yields $V-\bar{V} \in \mathcal{C}\left(\left[0, T_{1}\right] ; B_{2,1}^{\sigma}\right)$. Moreover it follows that $V_{t} \in \mathcal{C}\left(\left[0, T_{1}\right] ; B_{2,1}^{\sigma-1}\right)$, which implies the condition (3.6) immediately. Hence, the local existence part of solutions is complete.

Concerning the uniqueness, we set $\tilde{V}=V_{1}-V_{2}$, where $V_{1}$ and $V_{2}$ are two solutions to the system (1.5) subject to the same initial data, respectively. Then the error solution $\tilde{V}$ satisfies

$$
\begin{aligned}
& \tilde{A}^{0}\left(V_{1}\right) \tilde{V}_{t}+\sum_{j=1}^{d} \tilde{A}^{j}\left(V_{1}\right) \tilde{V}_{x_{j}} \\
& =-\tilde{A}^{0}\left(V_{1}\right) \sum_{j=1}^{d}\left\{\tilde{A}^{0}\left(V_{1}\right)^{-1} \tilde{A}^{j}\left(V_{1}\right)-\tilde{A}^{0}\left(V_{2}\right)^{-1} \tilde{A}^{j}\left(V_{2}\right)\right\} V_{2 x_{j}} .
\end{aligned}
$$

As above, following from the proof of Cauchy sequence, we obtain the inequality

$$
\|\tilde{V}\|_{\widetilde{L}_{T_{1}}^{\infty}\left(B_{2,1}^{\sigma-1}\right)} \leq C \int_{0}^{T_{1}}\left(\left\|V_{1}-\bar{V}\right\|_{B_{2,1}^{\sigma}}+\left\|V_{2}-\bar{V}\right\|_{B_{2,1}^{\sigma}}\right)\|\tilde{V}\|_{\widetilde{L}_{t}^{\infty}\left(B_{2,1}^{\sigma-1}\right)} d t .
$$

Gronwall's inequality implies $\tilde{V}=0$ immediately, i.e., the uniqueness of solution $V$ is achieved. The blow-up criterion follows from the works of Iftimie and Chae [20, 4] directly, we omit the details. This finishes the proof of Proposition 3.1 .

\section{$4 \quad$ A priori estimate and global existence}

To show that the classical solutions in Proposition 3.1 are globally defined, in this section, the central task is to construct further a priori estimates based on the dissipative mechanism produced by the source term.

To this end, we set

$$
E(T):=\|V-\bar{V}\|_{\widetilde{L}_{T}^{\infty}\left(B_{2,1}^{\sigma}\right)}
$$

and

$$
D(T):=\|(I-\mathcal{P}) V\|_{\widetilde{L}_{T}^{2}\left(B_{2,1}^{\sigma}\right)}+\|\nabla V\|_{\widetilde{L}_{T}^{2}\left(B_{2,1}^{\sigma-1}\right)}
$$

for any time $T>0$.

Our a priori estimate reads as follows.

Proposition 4.1. If $V-\bar{V} \in \widetilde{\mathcal{C}}_{T}\left(B_{2,1}^{\sigma}\right) \cap \widetilde{\mathcal{C}}_{T}^{1}\left(B_{2,1}^{\sigma-1}\right)$ is a solution of (1.5) and (3.1) for any $T>0$, then there exist some positive constants $\delta_{1}, \mu_{1}$ and $C_{1}$, if $E(T) \leq \delta_{1}$, then

$$
E(T)+\mu_{1} D(T) \leq C_{1}\left\|V_{0}-\bar{V}\right\|_{B_{2,1}^{\sigma}} .
$$

Thanks to the standard continuation argument, we extend the local-in-time solutions in Proposition 3.1 with the aid of Proposition 4.1, and obtain the global existence of classical solutions to the system (1.5) and (3.1), here we omit details, see, e.g., [28]. Then it follows from 
Theorem 1.1 and Proposition 2.3 that $U \in \mathcal{C}^{1}\left(\mathbb{R}^{+} \times \mathbb{R}^{d}\right)$ is the global-in-time classical solutions of (1.1)-(1.2). Furthermore, we arrive at Theorem 1.5,

Actually, the proof of Proposition 4.1 is to capture the dissipation rates from contributions of $(I-\mathcal{P}) V$ and $\nabla V$ in turn by using the frequency-localization method. For clarity, we divide it into several lemmas.

Lemma 4.1. If $V-\bar{V} \in \widetilde{\mathcal{C}}_{T}\left(B_{2,1}^{\sigma}\right) \cap \widetilde{\mathcal{C}}_{T}^{1}\left(B_{2,1}^{\sigma-1}\right)$ is a solution of (1.5) and (3.1) for any $T>0$, then the following estimate holds:

$$
\|V-\bar{V}\|_{L_{T}^{\infty}\left(L^{2}\right)}+\|(I-\mathcal{P}) V\|_{L_{T}^{2}\left(L^{2}\right)} \leq C\left\|V_{0}-\bar{V}\right\|_{L^{2}}
$$

where $C$ is a uniform positive constant independent of $T$.

Proof. It follows from Remark 2.2 and Lemma 2.2 that

$$
\sup _{0 \leq t \leq T}\|V(t, \cdot)-\bar{V}\|_{L^{\infty}} \leq C E(T) \leq C \delta_{1},
$$

so $V(t, x)$ takes values in a neighborhood of $\bar{V} \in \mathcal{M}$.

Let $\bar{U} \in \mathcal{E}$ be the constant state corresponding to $\bar{V} \in \mathcal{M}$. Denote the relative entropy function $\hat{\eta}(U)$ by

$$
\hat{\eta}(U):=\eta(U)-\eta(\bar{U})-\left\langle D_{U} \eta(\bar{U}), U-\bar{U}\right\rangle .
$$

Then the strictly convex quantity $\hat{\eta}(U)$ satisfies

$$
\hat{\eta}(U) \geq 0, \quad \hat{\eta}(\bar{U})=0, \quad D_{U} \hat{\eta}(\bar{U})=0 .
$$

Furthermore, $\hat{\eta}(U)$ is equivalent to the quadratic function $|U-\bar{U}|^{2}$ and hence to $|V-\bar{V}|^{2}$, since $\hat{\eta}(U)$ is strictly convex in $U$. On the other hand, from (1.1), we get the entropy-entropy flux equation

$$
\hat{\eta}(U)_{t}+\sum_{j=1}^{d} \hat{q}^{j}(U)_{x_{j}}=\left\langle D_{U} \eta(U), G(U)\right\rangle
$$

with $\hat{q}^{j}(U)(j=1, \cdots, d)=q^{j}(U)-q^{j}(\bar{U})-\left\langle D_{U} \eta(\bar{U}), F^{j}(U)-F^{j}(\bar{U})\right\rangle$, where $q^{j}(U)$ is the associated entropy flux with the entropy $\eta(U)$. It follows from (1.3) and Proposition 1.1 that

$$
\left\langle D_{U} \eta(U), G(U)\right\rangle=\langle W, H(W)\rangle \leq-C|(I-\mathcal{P}) W|^{2} \leq-C|(I-\mathcal{P}) V|^{2},
$$

where we used the fact that $W \in \mathcal{M}$ is equivalent to $V \in \mathcal{M}$. Integrating (4.5) over $[0, T] \times \mathbb{R}^{d}$ implies the desired inequality (4.2) immediately.

Lemma 4.2. If $V-\bar{V} \in \widetilde{\mathcal{C}}_{T}\left(B_{2,1}^{\sigma}\right) \cap \widetilde{\mathcal{C}}_{T}^{1}\left(B_{2,1}^{\sigma-1}\right)$ is a solution of (1.5) and (3.1) for any $T>0$, then the following estimate holds:

$$
\begin{aligned}
& \|V-\bar{V}\|_{\widetilde{L}_{T}^{\infty}\left(\dot{B}_{2,1}^{\sigma}\right)}+\|(I-\mathcal{P}) V\|_{\widetilde{L}_{T}^{2}\left(\dot{B}_{2,1}^{\sigma}\right)} \\
\leq & C\left\|V_{0}-\bar{V}\right\|_{\dot{B}_{2,1}^{\sigma}}+C \sqrt{\|V-\bar{V}\|_{\widetilde{L}_{T}^{\infty}\left(\dot{B}_{2,1}^{\sigma}\right)}}\left(\|\nabla V\|_{\widetilde{L}_{T}^{2}\left(\dot{B}_{2,1}^{\sigma-1}\right)}\right. \\
& \left.+\|(I-\mathcal{P}) V\|_{\widetilde{L}_{T}^{2}\left(\dot{B}_{2,1}^{\sigma-1}\right)}+\|(I-\mathcal{P}) V\|_{\widetilde{L}_{T}^{2}\left(\dot{B}_{2,1}^{\sigma}\right)}\right) .
\end{aligned}
$$

where $C$ is a uniform positive constant independent of $T$. 
Proof. Indeed, from Corollary 1.1, the normal form (1.5) can be written as

$$
\tilde{A}^{0}(V) V_{t}+\sum_{j=1}^{d} \tilde{A}^{j}(V) V_{x_{j}}+L V=\tilde{r}(V) .
$$

Applying the homogeneous localization operator $\dot{\Delta}_{q}(q \in \mathbb{Z})$ to (4.7), we obtain

$$
\tilde{A}^{0}(V) \dot{\Delta}_{q} V_{t}+\sum_{j=1}^{d} \tilde{A}^{j}(V) \dot{\Delta}_{q} V_{x_{j}}+L \dot{\Delta}_{q} V=I_{1}(V)+I_{2}(V)
$$

with

$$
I_{1}(V)=-\sum_{j=1}^{d} \tilde{A}^{0}(V)\left[\dot{\Delta}_{q}, \tilde{A}^{0}(V)^{-1} \tilde{A}^{j}(V)\right] V_{x_{j}}
$$

and

$$
I_{2}(V)=\tilde{A}^{0}(V)\left\{-\left[\dot{\Delta}_{q}, \tilde{A}^{0}(V)^{-1}\right] L V+\dot{\Delta}_{q}\left(\tilde{A}^{0}(V)^{-1} \tilde{r}(V)\right)\right\},
$$

where the commutator $[\cdot, \cdot]$ is defined by $[f, g]:=f g-g f$. Take the inner product of (4.8) with $\dot{\Delta}_{q} V$ to get

$$
\begin{array}{r}
\left\langle\tilde{A}^{0}(V) \dot{\Delta}_{q} V, \dot{\Delta}_{q} V\right\rangle_{t}+\sum_{j=1}^{d}\left\langle\tilde{A}^{j}(V) \dot{\Delta}_{q} V, \dot{\Delta}_{q} V\right\rangle_{x_{j}}+2\left\langle L \dot{\Delta}_{q} V, \dot{\Delta}_{q} V\right\rangle \\
=2\left\langle\left(I_{1}(V)+I_{2}(V), \dot{\Delta}_{q} V\right\rangle+\left\langle\operatorname{div} \mathbb{A}(V) \dot{\Delta}_{q} V, \dot{\Delta}_{q} V\right\rangle\right.
\end{array}
$$

where

$$
\operatorname{div} \mathbb{A}(V)=\tilde{A}^{0}(V)_{t}+\sum_{j=1}^{d} \tilde{A}^{j}(V)_{x_{j}}
$$

and

$$
\|f\|_{L_{\tilde{A}^{0}}^{2}}:=\left(\int_{\mathbb{R}^{d}}\left\langle\tilde{A}^{0}(V) f, f\right\rangle d x\right)^{1 / 2},\|f\|_{\widetilde{L}_{T}^{2}\left(\dot{\mathcal{B}}_{2,1}^{\sigma}\right)}=\left\|2^{q \sigma}\right\| \dot{\Delta}_{q} f\left\|_{L_{T}^{2}\left(L_{\tilde{A}^{0}}^{2}\right)}\right\|_{\ell^{1}(\mathbb{Z})} .
$$

Obviously, the norms $\|f\|_{L_{\tilde{A}^{0}}^{2}} \approx\|f\|_{L^{2}}$ and $\|f\|_{\widetilde{L}_{T}^{2}\left(\dot{\mathcal{B}}_{2,1}^{\sigma}\right)} \approx\|f\|_{\widetilde{L}_{T}^{2}\left(\dot{B}_{2,1}^{\sigma}\right)}$, since $V(t, x)$ takes values in a neighborhood of $\bar{V} \in \mathcal{M}$.

Integrating (4.9) over $[0, t] \times \mathbb{R}^{d}(t \in[0, T])$ gives

$$
\begin{aligned}
& \left.\left\|\dot{\Delta}_{q}(V-\bar{V})\right\|_{L_{\tilde{A}^{0}}^{2}}^{2}\right|_{0} ^{t}+\left\|(I-\mathcal{P}) \dot{\Delta}_{q} V\right\|_{L_{t}^{2}\left(L^{2}\right)}^{2} \\
= & C \int_{\mathbb{R}^{+}} \int_{\mathbb{R}^{d}}\left\langle I_{1}(V)+I_{2}(V), \dot{\Delta}_{q} V\right\rangle d x d t \\
& +C \int_{\mathbb{R}^{+}} \int_{\mathbb{R}^{d}}\left\langle\operatorname{div} \mathbb{A}(V) \dot{\Delta}_{q} V, \dot{\Delta}_{q} V\right\rangle d x d t,
\end{aligned}
$$

where we used the fact $\dot{\Delta}_{q} \bar{V}=0$. Next, we begin to estimate the integrations in the right-hand side of (4.10) respectively. 
From the equations (1.5) and Corollary 1.1, we have $\left|V_{t}\right| \leq C(|\nabla V|+|(I-\mathcal{P}) V|)$. Then

$$
\begin{aligned}
& \left|\int_{\mathbb{R}^{+}} \int_{\mathbb{R}^{d}}\left\langle\operatorname{div} \mathbb{A}(V) \dot{\Delta}_{q} V, \dot{\Delta}_{q} V\right\rangle d x d t\right| \\
\leq & C \int_{\mathbb{R}^{+}}\left(\left\|V_{t}\right\|_{L^{\infty}}+\|\nabla V\|_{L^{\infty}}\right)\left\|\dot{\Delta}_{q} V\right\|_{L^{2}}^{2} d t \\
\leq & C \int_{\mathbb{R}^{+}}\left(\|\nabla V\|_{L^{\infty}}+\|(I-\mathcal{P}) V\|_{L^{\infty}}\right)\left\|\dot{\Delta}_{q} V\right\|_{L^{2}}^{2} d t \\
\leq & C\left(\|\nabla V\|_{L_{t}^{2}\left(L^{\infty}\right)}+\|(I-\mathcal{P}) V\|_{L_{t}^{2}\left(L^{\infty}\right)}\right)\left\|\dot{\Delta}_{q} V\right\|_{L_{t}^{\infty}\left(L^{2}\right)}\left\|\dot{\Delta}_{q} V\right\|_{L_{t}^{2}\left(L^{2}\right)} \\
\leq & C c_{q}^{2} 2^{-2 q \sigma}\left(\|\nabla V\|_{L_{t}^{2}\left(\dot{B}_{2,1}^{\sigma-1}\right)}+\|(I-\mathcal{P}) V\|_{L_{t}^{2}\left(\dot{B}_{2,1}^{\sigma-1}\right)}\right) \\
& \times\|V-\bar{V}\|_{\widetilde{L}_{t}^{\infty}\left(\dot{B}_{2,1}^{\sigma}\right)}\|\nabla V\|_{\widetilde{L}_{t}^{2}\left(\dot{B}_{2,1}^{\sigma-1}\right)},
\end{aligned}
$$

where we used the embedding property (5) of Lemma 2.2. Here and below $\left\{c_{q}\right\}$ denotes some sequence which satisfies $\left\|\left(c_{q}\right)\right\|_{l^{1}(\mathbb{Z})} \leq 1$, although each $\left\{c_{q}\right\}$ is possibly different.

With the aid of Cauchy-Schwartz inequality and the commutator estimates in Proposition 2.4, we have

$$
\begin{aligned}
& \int_{\mathbb{R}^{+}} \int_{\mathbb{R}^{d}}\left\langle I_{1}(V), \dot{\Delta}_{q} V\right\rangle d x d t \\
\leq & C \sum_{j=1}^{d}\left\|\left[\dot{\Delta}_{q}, \tilde{A}^{0}(V)^{-1} \tilde{A}^{j}(V)\right] V_{x_{j}}\right\|_{L_{t}^{2}\left(L^{2}\right)}\left\|\dot{\Delta}_{q} V\right\|_{L_{t}^{2}\left(L_{\tilde{A}^{0}}^{2}\right)} \\
\leq & C c_{q} 2^{-q \sigma}\left\|\tilde{A}^{0}(V)^{-1} \tilde{A}^{j}(V)\right\|_{\widetilde{L}_{t}^{\infty}\left(\dot{B}_{2,1}^{\sigma}\right)}\|\nabla V\|_{\widetilde{L}_{t}^{2}\left(\dot{B}_{2,1}^{\sigma-1}\right)}\left\|\dot{\Delta}_{q} V\right\|_{L_{t}^{2}\left(L_{\tilde{A}^{0}}^{2}\right)} \\
\leq & C c_{q} 2^{-q \sigma}\|V-\bar{V}\|_{\widetilde{L}_{t}^{\infty}\left(\dot{B}_{2,1}^{\sigma}\right)}\|\nabla V\|_{\widetilde{L}_{t}^{2}\left(\dot{B}_{2,1}^{\sigma-1}\right)}\left\|\dot{\Delta}_{q} V\right\|_{L_{t}^{2}\left(L_{\tilde{A}^{0}}^{2}\right)}
\end{aligned}
$$

and

$$
\begin{aligned}
& \int_{\mathbb{R}^{+}} \int_{\mathbb{R}^{d}}\left\langle I_{2}(V), \dot{\Delta}_{q} V\right\rangle d x d t \\
\leq & C\left(\left\|\left[\dot{\Delta}_{q}, \tilde{A}^{0}(V)^{-1}\right] L V\right\|_{L_{t}^{2}\left(L^{2}\right)}+\left\|\dot{\Delta}_{q}\left(\tilde{A}^{0}(V)^{-1} \tilde{r}(V)\right)\right\|_{L_{t}^{2}\left(L^{2}\right)}\right) \\
& \times\left\|(I-\mathcal{P}) \dot{\Delta}_{q} V\right\|_{L_{t}^{2}\left(L_{\tilde{A}^{0}}^{2}\right)} \\
\leq & C c_{q} 2^{-q \sigma}\left(\left\|\tilde{A}^{0}(V)^{-1}\right\|_{\widetilde{L}_{t}^{\infty}\left(\dot{B}_{2,1}^{\sigma}\right)}\|L V\|_{\widetilde{L}_{t}^{2}\left(\dot{B}_{2,1}^{\sigma-1}\right)}+\left\|\tilde{A}^{0}(V)^{-1} \tilde{r}(V)\right\|_{\widetilde{L}_{t}^{2}\left(\dot{B}_{2,1}^{\sigma}\right)}\right) \\
& \times\left\|(I-\mathcal{P}) \dot{\Delta}_{q} V\right\|_{L_{t}^{2}\left(L_{\tilde{A}^{0}}^{2}\right)} \\
\leq & C c_{q} 2^{-q \sigma}\|V-\bar{V}\|_{\widetilde{L}_{t}^{\infty}\left(\dot{B}_{2,1}^{\sigma}\right)}\left(\|(I-P) V\|_{\widetilde{L}_{t}^{2}\left(\dot{B}_{2,1}^{\sigma-1}\right)}+\|\nabla V\|_{\widetilde{L}_{t}^{2}\left(\dot{B}_{2,1}^{\sigma-1}\right)}\right) \\
& \times\left\|(I-\mathcal{P}) \dot{\Delta}_{q} V\right\|_{L_{t}^{2}\left(L_{\tilde{A}^{0}}^{2}\right)},
\end{aligned}
$$

where we also used the homogeneous versions as Propositions 2.2 2.3.

Combining with (4.10)-(4.13), we are led to the estimate

$$
\left\|\dot{\Delta}_{q}(V-\bar{V})\right\|_{L_{\tilde{A}^{0}}^{2}}^{2}+\left\|(I-\mathcal{P}) \dot{\Delta}_{q} V\right\|_{L_{t}^{2}\left(L^{2}\right)}^{2}
$$




$$
\begin{aligned}
\leq & \left\|\dot{\Delta}_{q}\left(V_{0}-\bar{V}\right)\right\|_{L_{\widetilde{A}^{0}}^{2}}^{2}+C c_{q}^{2} 2^{-2 q \sigma}\left(\|\nabla V\|_{\widetilde{L}_{t}^{2}\left(\dot{B}_{2,1}^{\sigma-1}\right)}+\|(I-\mathcal{P}) V\|_{\widetilde{L}_{t}^{2}\left(\dot{B}_{2,1}^{\sigma-1}\right)}\right) \\
& \times\|V-\bar{V}\|_{\widetilde{L}_{t}^{\infty}\left(\dot{B}_{2,1}^{\sigma}\right)}\|\nabla V\|_{\widetilde{L}_{t}^{2}\left(\dot{B}_{2,1}^{\sigma-1}\right)}+C c_{q} 2^{-q \sigma}\|V-\bar{V}\|_{\widetilde{L}_{t}^{\infty}\left(\dot{B}_{2,1}^{\sigma}\right)}\|\nabla V\|_{\widetilde{L}_{t}^{2}\left(\dot{B}_{2,1}^{\sigma-1}\right)} \\
& \times\left\|\dot{\Delta}_{q} V\right\|_{L_{t}^{2}\left(L_{\tilde{A}^{0}}^{2}\right)}+C c_{q} 2^{-q \sigma}\|V-\bar{V}\|_{\widetilde{L}_{t}^{\infty}\left(\dot{B}_{2,1}^{\sigma}\right)}\left(\|(I-\mathcal{P}) V\|_{\widetilde{L}_{t}^{2}\left(\dot{B}_{2,1}^{\sigma-1}\right)}\right. \\
& \left.+\|\nabla V\|_{\widetilde{L}_{t}^{2}\left(\dot{B}_{2,1}^{\sigma-1}\right)}\right)\left\|(I-\mathcal{P}) \dot{\Delta}_{q} V\right\|_{L_{t}^{2}\left(L_{\tilde{A}^{0}}^{2}\right)} .
\end{aligned}
$$

Then multiplying the factor $2^{2 q \sigma}$ on both sides of (4.14), we obtain

$$
\begin{aligned}
& 2^{2 q \sigma}\left\|\dot{\Delta}_{q}(V-\bar{V})\right\|_{L_{\tilde{A}^{0}}^{2}}^{2}+2^{2 q \sigma}\left\|(I-\mathcal{P}) \dot{\Delta}_{q} V\right\|_{L_{t}^{2}\left(L^{2}\right)}^{2} \\
\leq & 2^{2 q \sigma}\left\|\dot{\Delta}_{q}\left(V_{0}-\bar{V}\right)\right\|_{L_{\tilde{A}^{0}}^{2}}^{2}+C c_{q}^{2}\left(\|\nabla V\|_{\widetilde{L}_{t}^{2}\left(\dot{B}_{2,1}^{\sigma-1}\right)}+\|(I-\mathcal{P}) V\|_{\widetilde{L}_{t}^{2}\left(\dot{B}_{2,1}^{\sigma-1}\right)}\right) \\
& \times\|V-\bar{V}\|_{\widetilde{L}_{t}^{\infty}\left(\dot{B}_{2,1}^{\sigma}\right)}\|\nabla V\|_{\widetilde{L}_{t}^{2}\left(\dot{B}_{2,1}^{\sigma-1}\right)}+C c_{q}^{2}\|V-\bar{V}\|_{\widetilde{L}_{t}^{\infty}\left(\dot{B}_{2,1}^{\sigma}\right)}\|\nabla V\|_{\widetilde{L}_{t}^{2}\left(\dot{B}_{2,1}^{\sigma-1}\right)} \\
& \times\|\nabla V\|_{\widetilde{L}_{t}^{2}\left(\dot{\mathcal{B}}_{2,1}^{\sigma-1}\right)}+C c_{q}^{2}\|V-\bar{V}\|_{\widetilde{L}_{t}^{\infty}\left(\dot{B}_{2,1}^{\sigma}\right)}\left(\|(I-\mathcal{P}) V\|_{\widetilde{L}_{t}^{2}\left(\dot{B}_{2,1}^{\sigma-1}\right)}\right. \\
& \left.+\|\nabla V\|_{\widetilde{L}_{t}^{2}\left(\dot{B}_{2,1}^{\sigma-1}\right)}\right)\|(I-\mathcal{P}) V\|_{\widetilde{L}_{t}^{2}\left(\dot{\mathcal{B}}_{2,1}^{\sigma}\right)}
\end{aligned}
$$

By performing Young's inequality, we have

$$
\begin{aligned}
& 2^{q \sigma}\left\|\dot{\Delta}_{q}(V-\bar{V})\right\|_{L_{T}^{\infty}\left(L^{2}\right)}+2^{q \sigma}\left\|(I-\mathcal{P}) \dot{\Delta}_{q} V\right\|_{L_{T}^{2}\left(L^{2}\right)} \\
\leq & 2^{q \sigma}\left\|\dot{\Delta}_{q}\left(V_{0}-\bar{V}\right)\right\|_{L^{2}}+C c_{q} \sqrt{\|V-\bar{V}\|_{\widetilde{L}_{T}^{\infty}\left(\dot{B}_{2,1}^{\sigma}\right)}} \\
& \times\left(\|\nabla V\|_{\widetilde{L}_{T}^{2}\left(\dot{B}_{2,1}^{\sigma-1}\right)}+\|(I-\mathcal{P}) V\|_{\widetilde{L}_{T}^{2}\left(\dot{B}_{2,1}^{\sigma-1}\right)}+\|(I-\mathcal{P}) V\|_{\widetilde{L}_{T}^{2}\left(\dot{B}_{2,1}^{\sigma}\right)}\right),
\end{aligned}
$$

where we used norms $\|f\|_{L_{\tilde{A}^{0}}^{2}} \approx\|f\|_{L^{2}}$ and $\|f\|_{\widetilde{L}_{T}^{2}\left(\dot{\mathcal{B}}_{2,1}^{\sigma}\right)} \approx\|f\|_{\widetilde{L}_{T}^{2}\left(\dot{B}_{2,1}^{\sigma}\right)}$.

Summing up (4.16) on $q \in \mathbb{Z}$, we can get (4.6) immediately.

Remark 4.1. According to the elementary fact

$$
L^{\theta}\left(L^{p}\right) \cap \widetilde{L}^{\theta}\left(\dot{B}_{p, r}^{s}\right)=\widetilde{L}^{\theta}\left(B_{p, r}^{s}\right)(\theta \geq r),
$$

and Corollary 6.1, which are achieved in the Appendix, it follows from (4.2) and (4.6) that

$$
E(T)+\|(I-\mathcal{P}) V\|_{\widetilde{L}_{T}^{2}\left(B_{2,1}^{\sigma}\right)} \leq C\left\|V_{0}-\bar{V}\right\|_{B_{2,1}^{\sigma}}+C \sqrt{E(T)} D(T) .
$$

Lemma 4.3. If $V-\bar{V} \in \widetilde{\mathcal{C}}_{T}\left(B_{2,1}^{\sigma}\right) \cap \widetilde{\mathcal{C}}_{T}^{1}\left(B_{2,1}^{\sigma-1}\right)$ is a solution of (1.5) and (3.1) for any $T>0$, then the following estimate holds:

$$
\begin{aligned}
\|\nabla V\|_{\widetilde{L}_{T}^{2}\left(B_{2,1}^{\sigma-1}\right)} \leq & C\left\|V_{0}-\bar{V}\right\|_{B_{2,1}^{\sigma}}+C E(T) \\
& +C\|(I-\mathcal{P}) V\|_{\widetilde{L}_{T}^{2}\left(B_{2,1}^{\sigma}\right)}+C \sqrt{E(T)} D(T),
\end{aligned}
$$

where $C$ is a uniform positive constant independent of $T$. 
Proof. In this paragraph, we shall take full advantage of the [SK] stability condition in Theorem 1.3 to establish the inequality (4.18).

For this purpose, we set $Z=V-\bar{V}$. Then the symmetric hyperbolic system (1.5) can be written as the linearized form at $\bar{V}$

$$
\tilde{A}^{0} Z_{t}+\sum_{j=1}^{d} \tilde{A}^{j} Z_{x_{j}}+L Z=\mathcal{G}
$$

where $\tilde{A}^{0}=\tilde{A}^{0}(\bar{V}), \tilde{A}^{j}=\tilde{A}^{j}(\bar{V})$ and $L$ are the constant matrices, and $\mathcal{G}=\mathcal{G}_{1}+\mathcal{G}_{2}$ with

$$
\begin{gathered}
\mathcal{G}_{1}:=-\sum_{j=1}^{d} \tilde{A}^{0}\left(\tilde{A}^{0}(V)^{-1} \tilde{A}^{j}(V)-\left(\tilde{A}^{0}\right)^{-1} \tilde{A}^{j}\right) V_{x_{j}}, \\
\mathcal{G}_{2}:=\tilde{A}^{0}\left\{-\left(\tilde{A}^{0}(V)^{-1}-\left(\tilde{A}^{0}\right)^{-1}\right) L V+\tilde{A}^{0}(V)^{-1} \tilde{r}(V)\right\} .
\end{gathered}
$$

Applying the inhomogeneous localization operator $\Delta_{q}(q \geq-1)$ to (4.19) gives

$$
\tilde{A}^{0} \Delta_{q} Z_{t}+\sum_{j=1}^{d} \tilde{A}^{j} \Delta_{q} Z_{x_{j}}+L \Delta_{q} Z=\Delta_{q} \mathcal{G} .
$$

Perform the Fourier transform with respect to the space variable $x$ for (4.20) and multiply the resulting equation by $-i|\xi| \tilde{K}(\omega)$. Then by taking the inner product with $\widehat{\Delta_{q} Z}$ and taking the real part of each term in the resulting equality, we can obtain

$$
\begin{gathered}
|\xi| \operatorname{Im}\left\langle\tilde{K}(\omega) \tilde{A}^{0} \frac{d}{d t} \widehat{\Delta_{q} Z}, \widehat{\Delta_{q} Z}\right\rangle+|\xi|^{2}\left\langle[\tilde{K}(\omega) \tilde{A}(\omega)]^{\prime} \widehat{\Delta_{q} Z}, \widehat{\Delta_{q} Z}\right\rangle \\
\quad=-|\xi| \operatorname{Im}\left\langle\tilde{K}(\omega) L \widehat{\Delta_{q} Z}, \widehat{\Delta_{q} Z}\right\rangle+|\xi| \operatorname{Im}\left\langle\tilde{K}(\omega) \widehat{\Delta_{q} \mathcal{G}}, \widehat{\Delta_{q} Z}\right\rangle
\end{gathered}
$$

with $\tilde{A}(\omega)=\sum_{j=1}^{d} \tilde{A}^{j} \omega_{j}$ and $\omega=\xi /|\xi|$.

The skew-symmetry of $\tilde{K}(\omega) \tilde{A}^{0}$ implies that

$$
|\xi| \operatorname{Im}\left\langle\tilde{K}(\omega) \tilde{A}^{0} \frac{d}{d t} \widehat{\Delta_{q} Z}, \widehat{\Delta_{q} Z}\right\rangle=\frac{1}{2} \frac{d}{d t}|\xi| \operatorname{Im}\left\langle\tilde{K}(\omega) \tilde{A}^{0} \widehat{\Delta_{q} Z}, \widehat{\Delta_{q} Z}\right\rangle .
$$

It follows from the condition that $[\tilde{K}(\omega) \tilde{A}(\omega)]^{\prime}+L$ is positive definite for $\omega \in \mathbb{S}^{d-1}$ that

$$
|\xi|^{2}\left\langle[\tilde{K}(\omega) \tilde{A}(\omega)]^{\prime} \widehat{\Delta_{q} Z}, \widehat{\Delta_{q} Z}\right\rangle \geq c|\xi|^{2}\left|\widehat{\Delta_{q} Z}\right|^{2}-C|\xi|^{2}\left|(I-\mathcal{P}) \widehat{\Delta_{q} Z}\right|^{2}
$$

Moreover, by virtue of Young's inequality, we have

$$
|| \xi\left|\operatorname{Im}\left\langle\tilde{K}(\omega) L \widehat{\Delta_{q} Z}, \widehat{\Delta_{q} Z}\right\rangle\right| \leq \varepsilon|\xi|^{2}\left|\widehat{\Delta_{q} Z}\right|^{2}+C_{\varepsilon}\left|(I-\mathcal{P}) \widehat{\Delta_{q} Z}\right|^{2}
$$

for any $\varepsilon>0$, and

$$
|| \xi\left|\operatorname{Im}\left\langle\tilde{K}(\omega) \widehat{\Delta_{q} \mathcal{G}}, \widehat{\Delta_{q} Z}\right\rangle\right| \leq C|\xi|\left|\widehat{\Delta_{q} Z}\right|\left|\widehat{\Delta_{q} \mathcal{G}}\right|
$$


where we have used the uniform boundedness of the matrix $\tilde{K}(\omega)$. Combining the equalities (4.21)-(4.22) and the inequalities (4.23)-(4.25), we deduce that

$$
\begin{aligned}
|\xi|^{2}\left|\widehat{\Delta_{q} Z}\right|^{2} \leq & C\left(1+|\xi|^{2}\right)\left|(I-\mathcal{P}) \widehat{\Delta_{q} Z}\right|^{2}+C|\xi|\left|\widehat{\Delta_{q} Z}\right|\left|\widehat{\Delta_{q} \mathcal{G}}\right| \\
& -\frac{1}{2} \frac{d}{d t}|\xi| \operatorname{Im}\left\langle\tilde{K}(\omega) \tilde{A}^{0} \widehat{\Delta_{q} Z}, \widehat{\Delta_{q} Z}\right\rangle .
\end{aligned}
$$

Integrating (4.26) over $[0, t] \times \mathbb{R}^{d}$, and using Plancherel's theorem yields

$$
\begin{aligned}
& \int_{0}^{t}\left\|\Delta_{q} \nabla Z\right\|_{L^{2}}^{2} d \tau \\
\leq & C \int_{0}^{t}\left(\left\|(I-\mathcal{P}) \Delta_{q} Z\right\|_{L^{2}}^{2}+\left\|(I-\mathcal{P}) \Delta_{q} \nabla Z\right\|_{L^{2}}^{2}\right) d \tau+C \int_{0}^{t}\left\|\Delta_{q} \nabla Z\right\|_{L^{2}}\left\|\Delta_{q} \mathcal{G}\right\|_{L^{2}} d \tau \\
& -\left.\frac{1}{2} \frac{d}{d t}|\xi| \operatorname{Im}\left\langle\tilde{K}(\omega) \tilde{A}^{0} \widehat{\Delta_{q} Z}, \widehat{\Delta_{q} Z}\right\rangle d \xi\right|_{0} ^{t} .
\end{aligned}
$$

The last term on the right side of (4.27) can be estimated as

$$
\begin{aligned}
& -\left.\frac{1}{2} \frac{d}{d t}|\xi| \operatorname{Im}\left\langle\tilde{K}(\omega) \tilde{A}^{0} \widehat{\Delta_{q} Z}, \widehat{\Delta_{q} Z}\right\rangle d \xi\right|_{0} ^{t} \\
\leq & C \int_{\mathbb{R}^{d}}\left(1+|\xi|^{2}\right)\left(\left|\left(\widehat{\Delta_{q} Z}\right)\right|^{2}+\left|\left(\widehat{\Delta_{q} Z_{0}}\right)\right|^{2}\right) d \xi \\
\leq & C 2^{2 q}\left(\left\|\Delta_{q} Z\right\|_{L^{2}}^{2}+\left\|\Delta_{q} Z_{0}\right\|_{L^{2}}^{2}\right),
\end{aligned}
$$

where we used Bernstein's inequality (Lemma 2.1) in the last step of (4.28). Substituting it into (4.27), we conclude that

$$
\begin{aligned}
& \left\|\Delta_{q} \nabla Z\right\|_{L_{t}^{2}\left(L^{2}\right)}^{2} \\
\leq & C 2^{2 q}\left(\left\|\Delta_{q} Z\right\|_{L_{T}^{\infty}\left(L^{2}\right)}^{2}+\left\|\Delta_{q} Z_{0}\right\|_{L^{2}}^{2}\right) \\
& +C\left(\left\|(I-\mathcal{P}) \Delta_{q} Z\right\|_{L_{T}^{2}\left(L^{2}\right)}^{2}+\left\|(I-\mathcal{P}) \Delta_{q} \nabla Z\right\|_{L_{T}^{2}\left(L^{2}\right)}^{2}\right) \\
& +C\left\|\Delta_{q} \nabla Z\right\|_{L_{T}^{2}\left(L^{2}\right)}\left\|\Delta_{q} \mathcal{G}\right\|_{L_{T}^{2}\left(L^{2}\right)} .
\end{aligned}
$$

Then multiplying the factor $2^{2 q(\sigma-1)}$ on both sides of (4.29) gives

$$
\begin{aligned}
& 2^{2 q(\sigma-1)}\left\|\Delta_{q} \nabla Z\right\|_{L_{t}^{2}\left(L^{2}\right)}^{2} \\
\leq & C c_{q}^{2}\left(\|Z\|_{\widetilde{L}_{T}^{\infty}\left(B_{2,1}^{\sigma}\right)}^{2}+\left\|Z_{0}\right\|_{B_{2,1}^{\sigma}}^{2}\right)+C c_{q}^{2}\left(\|(I-\mathcal{P}) Z\|_{\widetilde{L}_{T}^{2}\left(B_{2,1}^{\sigma}\right)}^{2}\right) \\
& +C c_{q}^{2}\|\nabla Z\|_{\widetilde{L}_{T}^{2}\left(B_{2,1}^{\sigma-1}\right)}\|\mathcal{G}\|_{\widetilde{L}_{T}^{2}\left(B_{2,1}^{\sigma-1}\right)}
\end{aligned}
$$

with $\|\mathcal{G}\|_{\widetilde{L}_{T}^{2}\left(B_{2,1}^{\sigma-1}\right)} \leq\left\|\mathcal{G}_{1}\right\|_{\widetilde{L}_{T}^{2}\left(B_{2,1}^{\sigma-1}\right)}+\left\|\mathcal{G}_{2}\right\|_{\widetilde{L}_{T}^{2}\left(B_{2,1}^{\sigma-1}\right)}$, where

$$
\left\|\mathcal{G}_{1}\right\|_{\widetilde{L}_{T}^{2}\left(B_{2,1}^{\sigma-1}\right)} \leq C\|Z\|_{\widetilde{L}_{T}^{\infty}\left(B_{2,1}^{\sigma-1}\right)}\|\nabla V\|_{\widetilde{L}_{T}^{2}\left(B_{2,1}^{\sigma-1}\right)}
$$

and

$$
\left\|\mathcal{G}_{2}\right\|_{\widetilde{L}_{T}^{2}\left(B_{2,1}^{\sigma-1}\right)} \leq\|Z\|_{\widetilde{L}_{T}^{\infty}\left(B_{2,1}^{\sigma-1}\right)}\|(I-\mathcal{P}) V\|_{\widetilde{L}_{T}^{2}\left(B_{2,1}^{\sigma-1}\right)}+C\left\|\tilde{A}^{0}(V)^{-1} \tilde{r}(V)\right\|_{\widetilde{L}_{T}^{2}\left(B_{2,1}^{\sigma-1}\right)}
$$


From Corollaries 6.1 and 1.1, we have

$$
\begin{aligned}
& \left\|\tilde{A}^{0}(V)^{-1} \tilde{r}(V)\right\|_{\widetilde{L}_{T}^{2}\left(B_{2,1}^{\sigma-1}\right)} \\
\leq & C\left(\left\|\tilde{A}^{0}(V)^{-1} \tilde{r}(V)\right\|_{L_{T}^{2}\left(L^{2}\right)}+\left\|\tilde{A}^{0}(V)^{-1} \tilde{r}(V)\right\|_{\widetilde{L}_{T}^{2}\left(\dot{B}_{2,1}^{\sigma-1}\right)}\right) \\
\leq & C\left(\|Z\|_{L_{T}^{\infty}\left(L^{\infty}\right)}\|(I-\mathcal{P}) V\|_{L_{T}^{2}\left(L^{2}\right)}+\left\|\tilde{A}^{0}(V)^{-1}\right\|_{\widetilde{L}_{T}^{\infty}\left(\dot{B}_{2,1}^{\sigma-1}\right)}\|\tilde{r}(V)\|_{\widetilde{L}_{T}^{2}\left(\dot{B}_{2,1}^{\sigma-1}\right)}\right) \\
\leq & C\left(\|Z\|_{\widetilde{L}_{T}^{\infty}\left(B_{2,1}^{\sigma-1}\right)}\|(I-\mathcal{P}) V\|_{\widetilde{L}_{T}^{2}\left(B_{2,1}^{\sigma}\right)}+\|Z\|_{\widetilde{L}_{T}^{\infty}\left(\dot{B}_{2,1}^{\sigma-1}\right)}\|\nabla V\|_{\widetilde{L}_{T}^{2}\left(\dot{B}_{2,1}^{\sigma-2}\right)}\right) \\
\leq & C\|Z\|_{\widetilde{L}_{T}^{\infty}\left(B_{2,1}^{\sigma-1}\right)}\left(\|(I-\mathcal{P}) V\|_{\widetilde{L}_{T}^{2}\left(B_{2,1}^{\sigma}\right)}+\|\nabla V\|_{\widetilde{L}_{T}^{2}\left(B_{2,1}^{\sigma-1}\right)}\right) .
\end{aligned}
$$

To conclude, we combine the estimates (4.30)-(4.33) to obtain

$$
\begin{aligned}
& 2^{2 q(\sigma-1)}\left\|\Delta_{q} \nabla Z\right\|_{L_{t}^{2}\left(L^{2}\right)}^{2} \\
\leq & C c_{q}^{2}\left(\|Z\|_{\widetilde{L}_{T}^{\infty}\left(B_{2,1}^{\sigma}\right)}^{2}+\left\|Z_{0}\right\|_{B_{2,1}^{\sigma}}^{2}\right)+C c_{q}^{2}\left(\|(I-\mathcal{P}) Z\|_{\widetilde{L}_{T}^{2}\left(B_{2,1}^{\sigma}\right)}^{2}\right) \\
& +C c_{q}^{2}\|\nabla Z\|_{\widetilde{L}_{T}^{2}\left(B_{2,1}^{\sigma-1}\right)}\left\{\|Z\|_{\widetilde{L}_{T}^{\infty}\left(B_{2,1}^{\sigma-1}\right)}\left(\|\nabla V\|_{\widetilde{L}_{T}^{2}\left(B_{2,1}^{\sigma-1}\right)}+\|(I-\mathcal{P}) V\|_{\widetilde{L}_{T}^{2}\left(B_{2,1}^{\sigma}\right)}\right)\right\} .
\end{aligned}
$$

By employing Young's inequality, we are led to the estimate

$$
\begin{aligned}
& 2^{q(\sigma-1)}\left\|\Delta_{q} \nabla Z\right\|_{L_{T}^{2}\left(L^{2}\right)} \\
\leq & C c_{q}\left(\|Z\|_{\widetilde{L}_{T}^{\infty}\left(B_{2,1}^{\sigma}\right)}+\left\|Z_{0}\right\|_{B_{2,1}^{\sigma}}\right)+C c_{q}\|(I-\mathcal{P}) Z\|_{\widetilde{L}_{T}^{2}\left(B_{2,1}^{\sigma}\right)} \\
& +C c_{q} \sqrt{\|Z\|_{\widetilde{L}_{T}^{\infty}\left(B_{2,1}^{\sigma}\right)}}\left(\|\nabla V\|_{\widetilde{L}_{T}^{2}\left(B_{2,1}^{\sigma-1}\right)}+\|(I-\mathcal{P}) V\|_{\widetilde{L}_{T}^{2}\left(B_{2,1}^{\sigma}\right)}\right) .
\end{aligned}
$$

In the end, summing up (4.35) on $q \geq-1$, we immediately deduce that

$$
\begin{aligned}
& \|\nabla Z\|_{\widetilde{L}_{T}^{2}\left(B_{2,1}^{\sigma-1}\right)} \\
\leq & C\left(\left\|Z_{0}\right\|_{B_{2,1}^{\sigma}}+E(T)\right)+C\|(I-\mathcal{P}) Z\|_{\widetilde{L}_{T}^{2}\left(B_{2,1}^{\sigma}\right)}+C \sqrt{E(T)} D(T),
\end{aligned}
$$

which is just the desired inequality (4.18).

The proof of Proposition 4.1. Combining the inequalities (4.17) and (4.18), we end up with

$$
\begin{aligned}
E(T)+D(T) & \leq C\left\|V_{0}-\bar{V}\right\|_{B_{2,1}^{\sigma}}+C \sqrt{E(T)} D(T) \\
& \leq C\left\|V_{0}-\bar{V}\right\|_{B_{2,1}^{\sigma}}+C \sqrt{\delta_{1}} D(T) .
\end{aligned}
$$

We take $\delta_{1}>0$ suitably small such that $C \sqrt{\delta_{1}} \leq 1 / 2$. Then the a priori estimate (4.1) follows readily. This finishes the proof of Proposition 4.1.

The proof of Corollary 1.2. From Remark 2.2 an the energy inequality (1.8), we get

$$
\nabla \mathcal{P} U \in L_{t}^{2}\left(B_{2,1}^{\sigma-1}\right) \quad \text { and } \quad \nabla \mathcal{P} U_{t} \in L_{t}^{2}\left(B_{2,1}^{\sigma-2}\right)
$$

for any $t>0$. Set

$$
\mathcal{H}(t)=\|\nabla \mathcal{P} U(\cdot, t)\|_{B_{2,1}^{\sigma-2}}^{2} \in L_{t}^{1}
$$


By direct calculations, it is not difficult to see that

$$
\frac{d}{d t} \mathcal{H}(t) \leqslant\|\nabla \mathcal{P} U(\cdot, t)\|_{B_{2,1}^{\sigma-2}}^{2}+\left\|\nabla \mathcal{P} U_{t}(\cdot, t)\right\|_{B_{2,1}^{\sigma-2}}^{2} \in L_{t}^{1} .
$$

Then apply to the embedding property in Sobolev spaces to get $\mathcal{H}(t) \rightarrow 0$, as $t \rightarrow+\infty$.

On the other hand, we can deduce that $\mathcal{P} U(t, x)-\bar{U}$ is bounded in $\mathcal{C}\left([0, \infty), B_{2,1}^{\sigma}\right)$. Hence, from interpolation arguments and the definition of projection operator $\mathcal{P}$, we obtain

$$
\|\nabla \mathcal{P}(U(\cdot, t)-\bar{U})\|_{B_{2,1}^{\sigma-1-\varepsilon}} \rightarrow 0(\varepsilon>0), \text { as } t \rightarrow+\infty .
$$

It further follows from Gagliardo-Nirenberg-Sobolev inequality in [12] that

$$
\|\mathcal{P} U(\cdot, t)-\bar{U}\|_{B_{p, 2}^{\sigma-1-\varepsilon}} \rightarrow 0 \quad\left(p=\frac{2 d}{d-2}, d>2\right) .
$$

What remains is the asymptotic behavior of $(I-\mathcal{P}) U$, which can be dealt with in a similar way, here, we omit the details.

\section{Applications}

In this section, we see that the new global existence result can be applied to some concrete partially dissipative hyperbolic equations, for instance, the compressible Euler equations with damping, which are given by the following form

$$
\left\{\begin{array}{l}
\partial_{t} \rho+\nabla \cdot(\rho \mathbf{u})=0 \\
\partial_{t}(\rho \mathbf{u})+\nabla \cdot(\rho \mathbf{u} \otimes \mathbf{u})+\nabla P=-\rho \mathbf{u}
\end{array}\right.
$$

Here $\rho=\rho(t, x)$ is the fluid density function of $(t, x) \in[0,+\infty) \times \mathbb{R}^{d}$ with $d \geq 1 ; \mathbf{u}=\left(u^{1}, u^{2}, \cdots\right.$ $\left.\cdot, u^{d}\right)^{\top}$ denotes the fluid velocity. The pressure $P$ is related to the density by $P(n)$, which satisfies

$$
P^{\prime}(\rho)>0, \quad \forall \rho>0 .
$$

For simplicity, a usual assumption is the $\gamma$-law: $P(\rho)=A \rho^{\gamma}(\gamma \geq 1)$, where $A>0$ is some physical constant, the adiabatic exponent $\gamma>1$ corresponds to the isentropic flow and $\gamma=1$ corresponds to the isothermal flow.

Let us consider the Cauchy problem of the compressible Euler equations (5.1) subject to the initial data

$$
(\rho, \mathbf{u})(0, x)=\left(\rho_{0}, \mathbf{u}_{0}\right) .
$$

The system (5.1) describes the compressible gas flow passes a porous medium and the medium induces a friction force, proportional to the linear momentum in the opposite direction. It is easy to show that (5.1) is a strict hyperbolic system when the smooth solutions $(\rho, \mathbf{u})$ away from vacuum. For the one-dimensional space case, the global existence of a smooth solution with small data was obtained first by Nishida [29], and the asymptotic behavior of the smooth solution was studied in many papers, see e.g. the excellent survey paper by Dafermos [9] and the book by Hsiao [16]. In multi-dimension space, as shown by [32, 34, if the initial data are small in some Sobolev space $H^{s}\left(\mathbb{R}^{d}\right)$ with $s>1+d / 2(s \in \mathbb{Z})$, the damping term can 
prevent the development of shock waves in finite time and the Cauchy problem (5.1)-(5.2) admits a unique global classical solution. Furthermore, it is proved that the solution in [32] has the $L^{\infty}$ convergence rate $(1+t)^{-3 / 2}(d=3)$ to the constant background state and the optimal $L^{p}(1<p \leq \infty)$ convergence rate $(1+t)^{-d / 2(1-1 / p)}$ in general several dimensions [34, respectively. For the large-time behavior of solutions with vacuum, the reader is referred to [18, 19].

Recently, in [14], Fang and the first author studied the existence and asymptotic behavior of classical solutions in the framework of Besov spaces with relatively lower regularity. From [14], we find that the low-frequency estimate of density for (5.1) is absent. Then, we overcame the difficulty by using Gagliardo-Nirenberg-Sobolev inequality (see, e.g., [12]) to obtain a global classical solution, regretfully, the result fails to hold in the critical Besov spaces $(\sigma=1+d / 2)$. In fact, we added a little regularity $(\sigma=1+d / 2+\varepsilon, \varepsilon>0)$ to ensure the global existence of classical solutions, which is essentially different from the Euler-Poisson equations in [35.

Now, to obtain the desired result of critical regularity, we hope that the compressible Euler equations (5.1) may enter in the class of the hyperbolic balance laws (1.1) of partially dissipative structure.

To this end, set

$$
U=\left(\begin{array}{c}
\rho \\
\mathbf{m}
\end{array}\right), \quad G(U)=\left(\begin{array}{c}
0 \\
-\mathbf{m}
\end{array}\right) \in \mathcal{M}^{\perp}
$$

with $\mathbf{m}=\rho \mathbf{u}$. Allow us to abuse the notations here. Moreover, corresponding to the orthogonal decomposition $\mathbb{R}^{d+1}=\mathcal{M} \oplus \mathcal{M}^{\perp}$ with $\operatorname{dim} \mathcal{M}=1$, we write $U=\mathcal{P} U+(I-\mathcal{P}) U$ with

$$
\mathcal{P} U=\left(\begin{array}{c}
\rho \\
0
\end{array}\right), \quad(I-\mathcal{P}) U=\left(\begin{array}{c}
0 \\
\mathbf{m}
\end{array}\right)
$$

where $\mathcal{P}$ is the orthogonal projection onto $\mathcal{M}$. In addition, the constant equilibrium state is $(\bar{\rho}, 0)^{\top} \in \mathcal{E}$.

Let us introduce the energy function

$$
\eta(U):=\frac{|\mathbf{m}|^{2}}{2 \rho}+h(\rho) \quad \text { with } \quad h^{\prime}(\rho)=\int_{1}^{\rho} \frac{P^{\prime}(s)}{s} d s .
$$

Define

$$
W=\left(\begin{array}{l}
W_{1} \\
W_{2}
\end{array}\right):=\nabla \eta(U)=\left(\begin{array}{c}
-\frac{|\mathbf{m}|^{2}}{2 \rho^{2}}+h^{\prime}(\rho) \\
\mathbf{m} / \rho
\end{array}\right) .
$$

Clearly, the mapping $U \rightarrow W$ is a diffeomorphism from $\mathcal{O}_{U}:=\mathbb{R}^{+} \times \mathbb{R}^{d}$ onto its range $\mathcal{O}_{W}$. Then for classical solutions $(\rho, \mathbf{u})$ away from vacuum, (5.1) is equivalent to the following system

$$
A^{0}(W) W_{t}+\sum_{j=1}^{d} A^{j}(W) W_{x_{j}}=H(W)
$$

with

$$
\begin{aligned}
& A^{0}(W)=\left(\begin{array}{cc}
1 & W_{2}^{\top} \\
W_{2} & W_{2} \otimes W_{2}+P^{\prime}(\rho) I_{d}
\end{array}\right) \\
& A^{j}(W)=\left(\begin{array}{cc}
W_{2 j} & W_{2}^{\top} W_{2 j}+P^{\prime}(\rho) e_{j}^{\top} \\
W_{2} W_{2 j}+P^{\prime}(\rho) e_{j} & W_{2 j}\left(W_{2} \otimes W_{2}+P^{\prime}(\rho) I_{d}\right)+P^{\prime}(\rho)\left(W_{2} \otimes e_{j}+e_{j} \otimes W_{2}\right)
\end{array}\right),
\end{aligned}
$$




$$
H(W)=G(U(W))=\left(\begin{array}{c}
0 \\
-P^{\prime}(\rho) W_{2}
\end{array}\right),
$$

where $I_{d}$ stands for the $d \times d$ unit matrix, and $e_{j}$ is $d$-dimensional vector where the $j$ th component is one, others are zero. It follows from the definition of variable $W$ that $h^{\prime}(\rho)=W_{1}+\left|W_{2}\right|^{2} / 2$, so $P^{\prime}(\rho)$ can be viewed as a function of $W$, since $\rho$ is the function of $W_{1}+\left|W_{2}\right|^{2} / 2$, i.e. of $W$.

It is not difficult to see that $A^{0}(W)$ is symmetric and positive definite, and the matrices $A^{j}(W)(j=1, \cdots, d)$ are symmetric. Obviously, $H(W)=0$ holds if and only if $W \in \mathcal{M}$. In addition,

$$
L(W)=-\nabla H(W)=\left(\begin{array}{cc}
0 & 0 \\
P^{\prime \prime}(\rho) \frac{\partial \rho}{\partial W_{1}} W_{2} & P^{\prime \prime}(\rho) \frac{\partial \rho}{\partial W_{2}} \otimes W_{2}+P^{\prime}(\rho) I_{d}
\end{array}\right) .
$$

For $W \in \mathcal{M}$, i.e. $W_{2}=0$, the matrix $L(W)$ is symmetric and nonnegative definite, and its null space coincides with $\mathcal{M}$. Hence, the system (5.3) is symmetric dissipative in the sense of Definition 1.2,

Set $\tilde{\eta}(W)=\rho h^{\prime}(\rho)-h(\rho)$, which can be viewed as the function of $W$. Then,

$$
D_{W} \tilde{\eta}(W)=\left(\begin{array}{c}
\frac{\partial \rho}{\partial W_{1}} h^{\prime}(\rho)+\rho-h^{\prime}(\rho) \frac{\partial \rho}{\partial W_{1}} \\
h^{\prime}(\rho) \frac{\partial \rho}{\partial W_{2}}+\rho W_{2}-h^{\prime}(\rho) \frac{\partial \rho}{\partial W_{2}}
\end{array}\right)=\left(\begin{array}{c}
\rho \\
\rho \cdot \frac{\mathbf{m}}{\rho}
\end{array}\right)=U,
$$

so it follows from the proof of Theorem[1.1] in 23 that $\langle U, W(U)\rangle-\tilde{\eta}(W)$ is an entropy function in the sense of Definition 1.1. By a straightforward computation, we find

$$
\langle U, W(U)\rangle-\tilde{\eta}(W)=(\rho, \mathbf{m})\left(\begin{array}{c}
-\frac{|\mathbf{m}|^{2}}{2 \rho^{2}}+h^{\prime}(\rho) \\
\mathbf{m} / \rho
\end{array}\right)-\left(\rho h^{\prime}(\rho)-h(\rho)\right)=\eta(U) .
$$

Hence, the energy function $\eta(U)$ is just the desired entropy. Furthermore, the associated entropy flux is

$$
q(U)=\left(\frac{|\mathbf{m}|^{2}}{2 \rho^{2}}+\rho h^{\prime}(\rho)\right) \frac{\mathbf{m}}{\rho} .
$$

Next, we show the linearized equations of the dissipative hyperbolic system (5.3) satisfies the stability condition, see Definition 1.4. Set $\bar{U}=(\bar{\rho}, 0)^{\top} \in \mathcal{E}$. The corresponding equilibrium state for the variable $W$ is $\bar{W}=\left(h^{\prime}(\bar{\rho}), 0\right)^{\top} \in \mathcal{M}$. The linearized equations near $\bar{W}=\left(h^{\prime}(\bar{\rho}), 0\right)^{\top}$ reads

$$
A^{0} W_{t}+\sum_{j=1}^{d} A^{j} W_{x_{j}}+L=0
$$

where

$$
\begin{gathered}
A^{0}=A^{0}(\bar{W})=\left(\begin{array}{cc}
1 & 0 \\
0 & P^{\prime}(\bar{\rho}) I_{d}
\end{array}\right), \\
A^{j}=A^{j}(\bar{W})=\left(\begin{array}{cc}
0 & P^{\prime}(\bar{\rho}) e_{j}^{\top} \\
P^{\prime}(\bar{\rho}) e_{j} & O
\end{array}\right), \\
L=L(\bar{W})=\left(\begin{array}{cc}
0 & 0 \\
0 & P^{\prime}(\bar{\rho}) I_{d}
\end{array}\right) .
\end{gathered}
$$


Claim: Let $\varphi=\left(\widetilde{W}_{1}, \widetilde{W}_{2}\right)^{\top} \in \mathbb{R}^{d+1}, \quad \lambda \in \mathbb{R}$ and $\omega \in \mathbb{S}^{d-1}$. If $L \varphi=0$ and $\lambda A^{0} \varphi+A(\omega) \varphi=0$, then $\varphi=0$.

Indeed, the condition $L \varphi=0$ gives $\widetilde{W}_{2}=0$. Then $\varphi=\left(\widetilde{W}_{1}, 0\right)^{\top}$. For this $\varphi$, we suppose that $\lambda A^{0} \varphi+A(\omega) \varphi=0$, which implies $\lambda \widetilde{W}_{1}=0$ and $P^{\prime}(\bar{\rho}) \widetilde{W}_{1} \omega=0$. This shows that $\widetilde{W}_{1}=0$ and hence $\varphi=0$.

Over all, the compressible Euler equations with damping are included in the class of dissipative hyperbolic equations and satisfy the stability condition at $\bar{W}$. Therefore, from Theorems 1.4 1.5, the Cauchy problem (5.1)-(5.2) admits the local classical solutions for general data in critical spaces.

Theorem 5.1. Let $\bar{\rho}>0$ be a constant reference density. Suppose that $\rho_{0}-\bar{\rho}$ and $\mathbf{m}_{0} \in B_{2,1}^{\sigma}$ with $\rho_{0}>0$, then there exists a time $T_{1}>0$ such that

(i) Existence: the Cauchy problem (5.1)-(5.2) has a unique solution $(\rho, \mathbf{m}) \in \mathcal{C}^{1}\left(\left[0, T_{1}\right] \times \mathbb{R}^{d}\right)$ with $\rho>0$ for all $t \in\left[0, T_{1}\right]$ and

$$
(\rho-\bar{\rho}, \mathbf{m}) \in \widetilde{\mathcal{C}}_{T_{1}}\left(B_{2,1}^{\sigma}\right) \cap \widetilde{\mathcal{C}}_{T_{0}}^{1}\left(B_{2,1}^{\sigma-1}\right) .
$$

(ii) Blow-up criterion: there exists a constant $C_{0}>0$ such that the maximal time $T^{*}$ of existence of such a solution can be bounded from below by $T^{*} \geq \frac{C_{0}}{\pi\left(\rho_{0}-\bar{\rho}, \mathbf{m}_{0}\right) \|_{B_{2,1}^{\sigma}}}$. Moreover, if $T^{*}$ is finite, then

$$
\limsup _{t \rightarrow T^{*}}\|(\rho-\bar{\rho}, \mathbf{m})\|_{B_{2,1}^{\sigma}}=\infty
$$

if and only if

$$
\int_{0}^{T^{*}}\|(\nabla \rho, \nabla \mathbf{m})\|_{L^{\infty}} d t=\infty
$$

Under the assumption of small initial data, we further obtain global-in-time classical solutions to (5.1)-(5.2) in critical spaces.

Theorem 5.2. Let $\bar{\rho}>0$ be a constant reference density. Suppose that $\rho_{0}-\bar{\rho}$ and $\mathbf{m}_{0} \in B_{2,1}^{\sigma}$, there exists a positive constant $\tilde{\delta}_{0}$ such that if

$$
\left\|\left(\rho_{0}-\bar{\rho}, \mathbf{m}_{0}\right)\right\|_{B_{2,1}^{\sigma}} \leq \tilde{\delta}_{0}
$$

with $\mathbf{m}_{0}=\rho_{0} \mathbf{u}_{0}$, then the Cauchy problem (5.1)-(5.2) has a unique global solution $(\rho, \mathbf{m})$ satisfying

$$
(\rho, \mathbf{m}) \in \mathcal{C}^{1}\left(\mathbb{R}^{+} \times \mathbb{R}^{d}\right)
$$

and

$$
(\rho-\bar{\rho}, \mathbf{m}) \in \widetilde{\mathcal{C}}\left(B_{2,1}^{\sigma}\right) \cap \widetilde{\mathcal{C}}^{1}\left(B_{2,1}^{\sigma-1}\right) .
$$

Furthermore, it holds that

$$
\begin{aligned}
& \|(\rho-\bar{\rho}, \mathbf{m})\|_{\widetilde{L}^{\infty}\left(B_{2,1}^{\sigma}\right)}+\tilde{\mu}_{0}\left(\|\mathbf{m}\|_{\widetilde{L}^{2}\left(B_{2,1}^{\sigma}\right)}+\|(\nabla \rho, \nabla \mathbf{m})\|_{\widetilde{L}^{2}\left(B_{2,1}^{\sigma-1}\right)}\right) \\
\leq & \tilde{C}_{0}\left\|\left(\rho_{0}-\bar{\rho}, \mathbf{m}_{0}\right)\right\|_{B_{2,1}^{\sigma}},
\end{aligned}
$$

where $\tilde{C}_{0}, \tilde{\mu}_{0}$ are some positive constants. 
Remark 5.1. In comparison with the previous works [32, 14, Theorem 5.1 indicates the critical regularity of global classical solutions. In addition, the result is valid for the general pressure in arbitrary space dimensions (Due to the techniques used, the papers [32, 14] were denoted to the system (5.1)-(5.2) with $\gamma$-law pressure in at least three space dimensions).

From Corollary 1.2, we also get the corresponding large-time behavior of $(\rho, \mathbf{m})$.

Corollary 5.1. Let $(\rho, \mathbf{m})$ be the solution in Theorem 5.1. Then

$$
\begin{gathered}
\|\nabla \rho(\cdot, t)\|_{B_{2,1}^{\sigma-1-\varepsilon}} \rightarrow 0, \\
\|\rho(\cdot, t)-\bar{\rho}\|_{B_{p, 2}^{\sigma-1-\varepsilon}} \rightarrow 0 \quad\left(p=\frac{2 d}{d-2}, d>2\right),
\end{gathered}
$$

and

$$
\|\mathbf{m}(\cdot, t)\|_{B_{2,1}^{\sigma-\varepsilon}} \rightarrow 0
$$

for any $\varepsilon>0$, as $t \rightarrow+\infty$.

\section{Appendix}

In this section, we first investigate the connection between the homogeneous Chemin-Lerner's spaces and inhomogeneous Chemin-Lerner's spaces. Precisely, we have

Proposition 6.1. Let $s>0,1 \leq \theta, p, r \leq \infty$. When $\theta \geq r$, it holds that

$$
L_{T}^{\theta}\left(L^{p}\right) \cap \widetilde{L}_{T}^{\theta}\left(\dot{B}_{p, r}^{s}\right)=\widetilde{L}_{T}^{\theta}\left(B_{p, r}^{s}\right)
$$

for any $T>0$.

Proof. Without loss of generality, we deal with $r<\infty$ only. In order to prove (6.1), we first show for all $s \in \mathbb{R}$

$$
L_{T}^{\theta}\left(L^{p}\right) \cap \widetilde{L}_{T}^{\theta}\left(\dot{B}_{p, r}^{s}\right) \subset \widetilde{L}_{T}^{\theta}\left(B_{p, r}^{s}\right) .
$$

Indeed, if $f \in L_{T}^{\theta}\left(L^{p}\right)$, then

$$
\left\|\Delta_{-1} f\right\|_{L_{T}^{\theta}\left(L^{p}\right)}=\|\Psi * f\|_{L_{T}^{\theta}\left(L^{p}\right)} \leq C\|f\|_{L_{T}^{\theta}\left(L^{p}\right)} .
$$

On the other hand, if $f \in \widetilde{L}_{T}^{\theta}\left(\dot{B}_{p, r}^{s}\right)$, then

$$
\left\{\sum_{q=-\infty}^{\infty}\left(2^{q s}\left\|\dot{\Delta}_{q} f\right\|_{L_{T}^{\theta}\left(L^{p}\right)}\right)^{r}\right\}^{1 / r}=\left\{\sum_{q=-\infty}^{\infty}\left(2^{q s}\left\|\Phi_{q} * f\right\|_{L_{T}^{\theta}\left(L^{p}\right)}\right)^{r}\right\}^{1 / r}<+\infty .
$$

Of course,

$$
\left\{\sum_{q=0}^{\infty}\left(2^{q s}\left\|\Phi_{q} * f\right\|_{L_{T}^{\theta}\left(L^{p}\right)}\right)^{r}\right\}^{1 / r}=\left\{\sum_{q=0}^{\infty}\left(2^{q s}\left\|\Delta_{q} f\right\|_{L_{T}^{\theta}\left(L^{p}\right)}\right)^{r}\right\}^{1 / r}<+\infty .
$$


Hence, we arrive at

$$
\left\{\sum_{q=-1}^{\infty}\left(2^{q s}\left\|\Delta_{q} f\right\|_{L_{T}^{\theta}\left(L^{p}\right)}\right)^{r}\right\}^{1 / r}<+\infty
$$

That is, $f \in \widetilde{L}_{T}^{\theta}\left(B_{p, r}^{s}\right)$.

Conversely, thanks to Remark $2.2(\theta \geq r)$ and $s>0$, we have the following imbeddings

$$
\widetilde{L}_{T}^{\theta}\left(B_{p, r}^{s}\right) \hookrightarrow L_{T}^{\theta}\left(B_{p, r}^{s}\right) \hookrightarrow L_{T}^{\theta}\left(B_{p, 1}^{0}\right) \hookrightarrow L_{T}^{\theta}\left(L^{p}\right),
$$

so if $f \in \widetilde{L}_{T}^{\theta}\left(B_{p, r}^{s}\right)$ then $f \in L_{T}^{\theta}\left(L^{p}\right)$.

In addition, for $q<0$, we obtain

$$
\left\|\dot{\Delta}_{q} f\right\|_{L_{T}^{\theta}\left(L^{p}\right)}=\left\{\begin{array}{l}
\left\|\Phi_{q} * \Psi * f\right\|_{L_{T}^{\theta}\left(L^{p}\right)} \leq C\left\|\Delta_{-1} f\right\|_{L_{T}^{\theta}\left(L^{p}\right)}, \quad q<-1, \\
\left\|\dot{\Delta}_{-1} f\right\|_{L_{T}^{\theta}\left(L^{p}\right)} \leq C\|f\|_{L_{T}^{\theta}\left(L^{p}\right)}, \quad q=-1 .
\end{array}\right.
$$

Hence, when $s>0$, it is not difficult to get

$$
\begin{aligned}
\|f\|_{\widetilde{L}_{T}^{\theta}\left(\dot{B}_{p, r}^{s}\right)} & =\left\{\sum_{q=-\infty}^{\infty}\left(2^{q s}\left\|\dot{\Delta}_{q} f\right\|_{L_{T}^{\theta}\left(L^{p}\right)}\right)^{r}\right\}^{1 / r} \\
& \leq\left\{\sum_{q<0}\left(2^{q s}\left\|\dot{\Delta}_{q} f\right\|_{L_{T}^{\theta}\left(L^{p}\right)}\right)^{r}\right\}^{1 / r}+\left\{\sum_{q \geq 0}\left(2^{q s}\left\|\dot{\Delta}_{q} f\right\|_{L_{T}^{\theta}\left(L^{p}\right)}\right)^{r}\right\}^{1 / r} \\
& \leq C \sum_{q<-1} 2^{q s}\left\|\dot{\Delta}_{q} f\right\|_{L_{T}^{\theta}\left(L^{p}\right)}+C 2^{-s}\left\|\dot{\Delta}_{-1} f\right\|_{L_{T}^{\theta}\left(L^{p}\right)}+\left\{\sum_{q \geq 0}\left(2^{q s}\left\|\dot{\Delta}_{q} f\right\|_{L_{T}^{\theta}\left(L^{p}\right)}\right)^{r}\right\}^{1 / r} \\
& \leq C\left\|\Delta_{-1} f\right\|_{L_{T}^{\theta}\left(L^{p}\right)}+\left\{\sum_{q \geq 0}\left(2^{q s}\left\|\Delta_{q} f\right\|_{L_{T}^{\theta}\left(L^{p}\right)}\right)^{r}\right\}^{1 / r}+C\|f\|_{L_{T}^{\theta}\left(L^{p}\right)} \\
& \leq C\|f\|_{\widetilde{L}_{T}^{\theta}\left(B_{p, r}^{s}\right)} .
\end{aligned}
$$

Therefore, $f \in L_{T}^{\theta}\left(L^{p}\right) \cap \widetilde{L}_{T}^{\theta}\left(\dot{B}_{p, r}^{s}\right)$ if $f \in \widetilde{L}_{T}^{\theta}\left(B_{p, r}^{s}\right)$.

Corollary 6.1. Let the assumptions of Proposition 6.1 be fulfilled. Then

$$
\|f\|_{\widetilde{L}_{T}^{\theta}\left(B_{p, r}^{s}\right)} \approx\|f\|_{L_{T}^{\theta}\left(L^{p}\right)}+\|f\|_{\widetilde{L}_{T}^{\theta}\left(\dot{B}_{p, r}^{s}\right)} .
$$

In what follows, we are concerned with the existence result for the linear problem (3.2)(3.3) in more general Besov spaces, which is used to establish the local existence in $B_{2,1}^{\sigma}$ for the quasilinear symmetric system (1.5) with (3.1).

Proposition 6.2. Let $T>0$ and let $r \in[1, \infty), s>0$ and $V_{0}-\bar{V} \in B_{2, r}^{s}$. Assume that

$$
\begin{gathered}
V-\bar{V} \in\left\{\begin{array}{l}
\mathcal{C}_{T}\left(B_{2, r}^{s}\right) \cap \mathcal{C}_{T}^{1}\left(B_{2, r}^{s-1}\right) \text { if } s>1+d / 2, \text { or } s=1+d / 2 \text { and } r=1 ; \\
\mathcal{C}_{T}\left(B_{2, \infty}^{s+\varepsilon}\right) \cap \mathcal{C}_{T}^{1}\left(B_{2, \infty}^{s-1+\varepsilon}\right) \text { for } \varepsilon>0 \text { if } s=1+d / 2 \text { and } r>1 ; \\
\mathcal{C}_{T}\left(B_{2, \infty}^{1+d / 2} \cap W^{1, \infty}\right) \cap \mathcal{C}_{T}^{1}\left(B_{2, \infty}^{d / 2} \cap L^{\infty}\right) \text { if } 0<s<1+d / 2 ;
\end{array}\right. \\
V(t, x) \in \mathcal{O}_{1} \quad \text { for any }(t, x) \in Q_{T},
\end{gathered}
$$


where $\mathcal{O}_{1}$ is a bounded open convex set in $\mathbb{R}^{N}$ satisfying $\overline{\mathcal{O}}_{1} \subset \mathcal{O}_{V}$. Then the system (3.2)-(3.3) has a unique solution $\hat{V}$ belongs to

$$
\hat{V}-\bar{V} \in \widetilde{\mathcal{C}}_{T}\left(B_{2, r}^{s}\right) \cap \widetilde{\mathcal{C}}_{T}^{1}\left(B_{2, r}^{s-1}\right)
$$

and satisfies

$$
\|\hat{V}-\bar{V}\|_{\widetilde{L}_{T}^{\infty}\left(B_{2, r}^{s}\right)} \leq\left\|V_{0}-\bar{V}\right\|_{B_{2, r}^{s}} e^{C \int_{0}^{T}\left(a_{1}(t)+a_{2}(t)\right) d t}
$$

where

$$
\begin{gathered}
a_{1}(t)=\left\|V_{t}(t, \cdot)\right\|_{L^{\infty}}, \\
a_{2}(t)=\left\{\begin{array}{l}
\|V(t, \cdot)-\bar{V}\|_{B_{2, r}^{s}} \text { if } s>1+d / 2, \text { or } s=1+d / 2 \text { and } r=1 ; \\
\|V(t, \cdot)-\bar{V}\|_{B_{2, \infty}^{s+\varepsilon}} \text { for } \varepsilon>0 \text { if } s=1+d / 2 \text { and } r>1 ; \\
\|V(t, \cdot)-\bar{V}\|_{B_{2, \infty}^{1+d / 2} \cap W^{1, \infty}} \text { if } 0<s<1+d / 2 .
\end{array}\right.
\end{gathered}
$$

Proof. The energy inequality (6.2) can follow from the proof of Lemma 3.1 at a similar way, however, we should point out the estimates of commutator $\left\|\left[\Delta_{q}, \tilde{A}^{0}(V)^{-1} \tilde{A}^{j}(V)\right] \hat{Z}_{x_{j}}\right\|_{L^{2}}$ for general indexes $s, r$. Precisely, from [2], we have

Lemma 6.1. For all $t \in[0, T]$ and $s>0$, it holds that

$$
\begin{aligned}
& 2^{q s}\left\|\left[\Delta_{q}, \tilde{A}^{0}(V)^{-1} \tilde{A}^{j}(V)\right] \hat{Z}_{x_{j}}\right\|_{L^{2}} \\
& \leq\left\{\begin{array}{l}
C c_{q}\left\|\nabla\left(\tilde{A}^{0}(V)^{-1} \tilde{A}^{j}(V)\right)\right\|_{B_{2, r}^{s-1}}\|\hat{Z}\|_{B_{2, r}^{s}}, \text { if } s>1+d / 2, \text { or } s=1+d / 2 \text { and } r=1 ; \\
C c_{q}\left\|\nabla\left(\tilde{A}^{0}(V)^{-1} \tilde{A}^{j}(V)\right)\right\|_{B_{2, \infty}^{s-1+\varepsilon}}\|\hat{Z}\|_{B_{2, r}^{s}}, \text { for } \varepsilon>0 \text { if } s=1+d / 2 \text { and } r>1 ; \\
C c_{q}\left\|\nabla\left(\tilde{A}^{0}(V)^{-1} \tilde{A}^{j}(V)\right)\right\|_{B_{2, \infty}^{d / 2} \cap L^{\infty}}\|\hat{Z}\|_{B_{2, r}^{s}}, \text { if } 0<s<1+d / 2,
\end{array}\right.
\end{aligned}
$$

where $\left\|c_{q}(t)\right\|_{\ell^{1}} \leq 1$, for all $t \in[0, T]$.

Note that these facts, similar to the steps $(\underline{3.9})-(3.16)$, we readily deduce that

$$
\begin{aligned}
\left\|\Delta_{q} \hat{Z}\right\|_{L_{t}^{\infty}\left(L^{2}\right)} \leq & \left\|\Delta_{q} \hat{Z}_{0}\right\|_{L^{2}}+C \int_{0}^{t} c_{q}(\tau) 2^{-q s} a_{2}(\tau)\|\hat{Z}\|_{B_{2, r}^{s}} d \tau \\
& +C \int_{0}^{t}\|\operatorname{div} \mathbb{A}(V)\|_{L^{\infty}}\left\|\Delta_{q} \hat{Z}\right\|_{L^{2}} d \tau .
\end{aligned}
$$

Then we multiply both sides by $2^{q s}$ and take the $\ell^{r}$ norm to obtain

$$
\|\hat{Z}\|_{\widetilde{L}_{T}^{\infty}\left(B_{2, r}^{s}\right)} \leq\left\|\hat{Z}_{0}\right\|_{B_{2, r}^{s}}+C \int_{0}^{T}\left(a_{1}(t)+a_{2}(t)\right)\|\hat{Z}\|_{\widetilde{L}_{t}^{\infty}\left(B_{2, r}^{s}\right)} d t .
$$

Applying Gronwall's inequality leads to the inequality (6.2) directly.

To show the existence of solution $\hat{V}(t, x)$, we use the classical Friedrichs' regularization method, which was used in [7] for example. More precisely, we consider the approximate system for $\hat{Z}_{k}=\hat{V}_{k}-\bar{V}$ :

$$
\partial_{t} \hat{Z}_{k}+\sum_{j=1}^{d} \tilde{A}^{0}(V)^{-1} \mathbb{P}_{k}\left(\tilde{A}^{j}(V) \partial_{x_{j}} \hat{Z}_{k}\right)=0
$$


with

$$
\left.\hat{Z}_{k}\right|_{t=0}=\mathbb{P}_{k} \hat{Z}_{0}
$$

where $\hat{Z}_{0}=\hat{V}_{0}-\bar{V}$ and $\mathbb{P}_{k}$ is the cut-off operator on $L^{2}\left(\mathbb{R}^{d}\right)$ defined by

$$
\mathbb{P}_{k} f:=\mathcal{F}^{-1}\left(\mathbf{1}_{B(0, k)} \mathcal{F} f\right)
$$

Denote the space

$$
L_{k}^{2}:=\left\{f \in L^{2}\left(\mathbb{R}^{d}\right): \operatorname{supp} \mathcal{F} f \subset B(0, k)\right\},
$$

where $B(0, k)$ is the ball with center 0 and radius $k$.

From Lemma 2.1, we can see that the operator $\partial_{x_{j}}$ is continuous on $L_{k}^{2}$. Furthermore, it turns out that the linear operator

$$
\hat{Z} \mapsto \sum_{j=1}^{d} \tilde{A}^{0}(V)^{-1} \mathbb{P}_{k}\left(\tilde{A}^{j}(V) \partial_{x_{j}} \hat{Z}\right)
$$

is also continuous on $L_{k}^{2}$, since the functions $\tilde{A}^{0}(V)^{-1}$ and $\tilde{A}^{j}(V)$ are both bounded in $[0, T] \times \mathbb{R}^{d}$. Thus, the approximate system (6.4) appears to a linear system of ordinary differential equations in $L_{k}^{2}$, which implies the existence of a unique function $\hat{V}_{k}(t, x)$ such that $\hat{Z}_{k}(t, x)=\hat{V}_{k}(t, x)-\bar{V} \in$ $\mathcal{C}^{1}\left([0, T], L_{k}^{2}\right)$ is the solution of (6.4)-(6.5) . Furthermore, it follows from the spectral localization that $\hat{Z}_{k}(t, x) \in \mathcal{C}^{1}\left([0, T], B_{2, r}^{\alpha}\right)$ for any $\alpha \in \mathbb{R}$.

Using the facts that the operator $\mathbb{P}_{k}$ is self-adjoint on $L^{2}$ and $\mathbb{P}_{k} \hat{Z}_{k}=\hat{Z}_{k}$, we proceed exactly as in the proof of the inequality (6.2) and obtain

$$
\begin{aligned}
\sup _{t \in[0, T]}\left\|\hat{Z}_{k}(t)\right\|_{B_{2, r}^{s}} & \leq\left\|\mathbb{P}_{k} \hat{Z}_{0}\right\|_{B_{2, r}^{s}} e^{C \int_{0}^{T}\left(a_{1}(t)+a_{2}(t)+a_{2}^{2}(t)\right) d t} \\
& \leq C\left\|V_{0}-\bar{V}\right\|_{B_{2, r}^{s}} e^{C \int_{0}^{T}\left(a_{1}(t)+a_{2}(t)+a_{2}^{2}(t)\right) d t} \\
& \leq C .
\end{aligned}
$$

Here and below, the constant $C>0$ independent of $k$. Furthermore, it follows from (6.4) and (6.6) that

$$
\sup _{t \in[0, T]}\left\|\partial_{t} \hat{Z}_{k}\right\|_{B_{2, r}^{s-1}} \leq C
$$

Therefore, we deduce that the approximative solution sequence $\left\{\hat{Z}_{k}=\hat{V}_{k}-\bar{V}\right\}$ to (6.4)(6.5) is uniformly bounded in $\mathcal{C}\left([0, T], B_{2, r}^{s}\right) \cap \mathcal{C}^{1}\left([0, T], B_{2, r}^{s-1}\right)$. Moreover, it weak ${ }^{\star}$-converges (up to a subsequence) to some function $\hat{V}$ such that $\hat{V}-\bar{V} \in L^{\infty}\left([0, T], B_{2, r}^{s}\right)$ in terms of the Banach-Alaoglu Theorem (see [33], Remark 2, p.180). Since $\left\{\partial_{t} \hat{V}_{k}\right\}$ is also uniformly bounded in $\mathcal{C}\left([0, T], B_{2, r}^{s-1}\right)$ (it weak ${ }^{\star}$-converges to $\hat{V}_{t}$ in $L^{\infty}\left([0, T], B_{2, r}^{s-1}\right)$, then $\left\{\hat{V}_{k}-\bar{V}\right\}$ is uniformly bounded in $\operatorname{Lip}\left([0, T], B_{2, r}^{s-1}\right)$, hence uniformly equicontinuous on $[0, T]$ with the norm in $B_{2, r}^{s-1}$. From Proposition 2.1, Ascoli-Arzela theorem and Cantor diagonal process, we arrive at

$$
\phi_{j}\left(\hat{V}_{k}-\bar{V}\right) \rightarrow \phi_{j}(\hat{V}-\bar{V}) \quad \text { in } \mathcal{C}\left([0, T], B_{2, r}^{s-1}\right)
$$


as $k \rightarrow \infty$, for $\phi_{j} \in C_{c}^{\infty}$ which is supported in the ball $B(0 ; j+1)$ and equal to 1 on $B(0 ; j)$. The property of strong convergence and

$$
\lim _{n \rightarrow \infty} \mathbb{P}_{k}\left(V_{0}-\bar{V}\right)=V_{0}-\bar{V} \text { in } \quad B_{2, r}^{s}
$$

enable us to pass to the limit in (6.4)-(6.5) and $\hat{V}$ is the solution of (3.2)-(3.3) in the sense of distribution. Next, we check the solution $\hat{V}$ has the required regularity. Indeed, $\hat{V}-\bar{V} \in$ $\mathcal{C}\left([0, T], B_{2, r}^{s-1}\right)$. On the other hand, since $\hat{V}-\bar{V} \in L^{\infty}\left([0, T], B_{2, r}^{s}\right)$, we have

$$
\left\|2^{q s}\right\| \Delta_{q}(\hat{V}-\bar{V})\left\|_{L^{2}}\right\|_{\ell^{r}}<+\infty .
$$

The inequality (6.10) implies that there exists an integer $q_{0}$ such that

$$
\left\{\sum_{q \geq q_{0}}\left(2^{q s}\left\|\Delta_{q}(\hat{V}-\bar{V})\right\|_{L^{2}}\right)^{r}\right\}^{1 / r}<\frac{\varepsilon}{4}
$$

for any positive constant $\varepsilon$. Then, we have

$$
\begin{aligned}
& \left\|\hat{V}(t)-\hat{V}\left(t^{\prime}\right)\right\|_{B_{2, r}^{s}} \\
\leq & \left\{\sum_{q<q_{0}}\left(2^{q s}\left\|\Delta_{q}\left(\hat{V}(t)-\hat{V}\left(t^{\prime}\right)\right)\right\|_{L^{2}}\right)^{r}\right\}^{1 / r}+2\left\{\sum_{q \geq q_{0}}\left(2^{q s}\left\|\Delta_{q}(\hat{V}(t)-\bar{V})\right\|_{L^{2}}\right)^{r}\right\}^{1 / r} \\
\leq & C \sum_{q<q_{0}} 2^{q s}\left\|\Delta_{q}\left(\hat{V}(t)-\hat{V}\left(t^{\prime}\right)\right)\right\|_{L^{2}}+\frac{\varepsilon}{2} \\
\leq & C 2^{q_{0} s}\left\|\hat{V}(t)-\hat{V}\left(t^{\prime}\right)\right\|_{L^{2}}+\frac{\varepsilon}{2} .
\end{aligned}
$$

Since $\hat{V}-\bar{V} \in \mathcal{C}\left([0, T], B_{2, r}^{s-1}\right) \hookrightarrow \mathcal{C}\left([0, T], L^{2}\right)$ for the case of $s>1$, the first term on the righthand side of (6.12) tends to zero where $t^{\prime}$ goes to $t$. This implies that $\hat{V}-\bar{V}$ is continuous in time with values in $B_{2, r}^{s}$. Using the fact that $\hat{V}$ is a solution of (3.2)-(3.3), we further conclude that $\hat{V}-\bar{V} \in \mathcal{C}\left([0, T], B_{2, r}^{s}\right) \cap \mathcal{C}^{1}\left([0, T], B_{2, r}^{s-1}\right)$. In the case where $0<s \leq 1$, we consider the regularized system by smoothing out the initial data:

$$
\left\{\begin{array}{l}
\tilde{A}^{0}(V) \partial_{t} \hat{Z}_{k}+\sum_{j=1}^{d} \tilde{A}^{j}(V) \partial_{x_{j}} \hat{Z}_{k}=0 \\
\left.\hat{Z}_{k}\right|_{t=0}=\mathbb{P}_{k} \hat{Z}_{0}
\end{array}\right.
$$

Thanks to the above result for the case of $s>1$, the solution $\hat{Z}_{k}=\hat{V}_{k}-\bar{V}$ of (6.13) is well defined on $[0, T]$ and belongs to $\mathcal{C}\left([0, T], B_{2, r}^{\alpha}\right) \cap \mathcal{C}^{1}\left([0, T], B_{2, r}^{\alpha-1}\right)$ for any $\alpha>1$. Furthermore, the function $\delta \hat{Z}_{k}:=\hat{Z}_{k+1}-\hat{Z}_{k}$ satisfies

$$
\left\{\begin{array}{l}
\tilde{A}^{0}(V) \partial_{t} \delta \hat{Z}_{k}+\sum_{j=1}^{d} \tilde{A}^{j}(V) \partial_{x_{j}} \delta \hat{Z}_{k}=0 \\
\left.\delta \hat{Z}_{k}\right|_{t=0}=\left(\mathbb{P}_{k+1}-\mathbb{P}_{k}\right) \hat{Z}_{0}
\end{array}\right.
$$

Similar to (6.2), we have

$$
\sup _{t \in[0, T]}\left\|\delta \hat{Z}_{k}(t)\right\|_{B_{2, r}^{s}} \leq\left\|\left(\mathbb{P}_{k+1}-\mathbb{P}_{k}\right) \hat{Z}_{0}\right\|_{B_{2, r}^{s}} e^{C \int_{0}^{T}\left(a_{1}(t)+a_{2}(t)\right) d t} .
$$


As $\hat{Z}_{0}=\hat{V}_{0}-\bar{V}$ belongs to $B_{2, r}^{s}(0<s \leq 1)$, the sequence $\left(\mathbb{P}_{k} \hat{Z}_{0}\right)_{k \in \mathbb{N}}$ converges to $\hat{Z}_{0}$. Thus, it follows from (6.15) that the sequence $\left(\hat{Z}_{k}\right)_{k \in \mathbb{N}}$ is Cauchy in $\mathcal{C}\left([0, T], B_{2, r}^{s}\right)$ and converges to some $\hat{Z}=\hat{V}-\bar{V} \in \mathcal{C}\left([0, T], B_{2, r}^{s}\right) \cap \mathcal{C}^{1}\left([0, T], B_{2, r}^{s-1}\right)$ in (6.13) , which is, of course, a solution of (3.2)- (3.3) .

Over all, we conclude that $\hat{V}(x, t)$ is the solution of (3.2)- (3.3) satisfying $\hat{V}-\bar{V} \in \mathcal{C}\left([0, T], B_{2, r}^{s}\right)$ $\cap \mathcal{C}^{1}\left([0, T], B_{2, r}^{s-1}\right)$ for $s>0$, furthermore, we arrive at $\hat{V}-\bar{V} \in \widetilde{\mathcal{C}}_{T}\left(B_{2, r}^{s}\right) \cap \widetilde{\mathcal{C}}_{T}^{1}\left(B_{2, r}^{s-1}\right)$.

Finally, the uniqueness is merely a consequence of (6.2). This completes the proof of Proposition 6.2

\section{Acknowledgments}

J. Xu is partially supported by the NSFC (11001127), China Postdoctoral Science Foundation (20110490134) and Postdoctoral Science Foundation of Jiangsu Province (1102057C). He would like to thank Professor Kawashima for his enthusiastic communication and hospitality. The second author (S. K.) is partially supported by Grant-in-Aid for Scientific Research (A) 22244009.

\section{References}

[1] D. Aregba-driollet and R. Natalini. Discrete kinetic schemes for multidimensional systems of conservation laws. SIAM J. Numer. Anal. 37 (2000) 1973-2004.

[2] H. Bahouri, J. Y. Chemin and R. Danchin. Fourier Analysis and Nonlinear Partial Differential Equations, Springer-Verlag: Berlin/Heidelberg, 2011.

[3] S. Bianchini, B. Hanouzet and R. Natalini. Asymptotic behavior of smooth solutions for partially dissipative hyperoblic systems with a convex entropy, Comm. Pure and Appl. Math. 60 (2007) 1559-1622.

[4] D. Chae. On the system of conservation laws and its perturbation in the Besov spaces. Ad. Diff. Eqns 10 (2005) 983-1006.

[5] D. Chae. On the well-posedness of the Euler equations in the Triebel-Lizorkin spaces, Comm. Pure and Appl. Math. 55 (2002) 654-678.

[6] J.-Y. Chemin. Théorèmes d'unicité pour le système de Navier-Stokes tridimensionnel, Journal d'Analyse Mathématique, 77 (1999), 27-50.

[7] J.-Y. Chemin. Localization in Fourier space and Navier-Stokes system, in: Phase Space Analysis of Partial Differential Equations, Proceedings 2004, CRM Series, 53-136.

[8] G.-Q. Chen, C. D. Levermore and T.-P. Liu. Hyperbolic conservation laws with stiff relaxation terms and entropy. Comm. Pure Appl. Math. 47 (1994) 787-830.

[9] C. M. Dafermos. Can dissipation prevent the breaking of waves? In: Transactions of the Twenty-Sixth Conference of Army Mathematicians, 187-198, ARO Rep. 81, 1, U. S. Army Res. Office, Research Triangle Park, N.C., 1981. 
[10] C. M. Dafermos. Hyperbolic conservation laws in continuum physics (Third Editor). Springer-Verlag: Berlin/Heidelberg, 2010.

[11] R. Danchin. Local theory in the critical spaces for compressible viscous and heat-conductive gases, Comm. P. D. E., 26 (2001) 1183-1233.

[12] L. C. Evans, Partial differential equations, Providence, Rhode Island: Amer Mathematical Society, 1998.

[13] K. O. Friedrichs and P. D. Lax. Systems of conservation equations with a convex exttension. Proc. Nat. Acad. Sci. USA 68 (1971) 1686-1688.

[14] D. Y. Fang and J. Xu. Existence and asymptotic behavior of $\mathcal{C}^{1}$ solutions to the multidimensional compressible Euler equations with damping, Nonlinear Anal. TMA 70 (2009) 244-261.

[15] S. K. Godunov. An interesting class of quasilinear systems. Dokl. Akad. Nauk SSSR 139 (1961) 521-523.

[16] L. Hsiao. Quasilinear Hyperbolic Systems and Dissipative Mechanisms, Singapore: World Scientific Publishing, 1997.

[17] B. Hanouzet and R. Natalini. Global existence of smooth solutions for partially disipative hyperbolic systems with a convex entropy. Arch. Rational Mech. Anal. 169 (2003) 89-117.

[18] F. Huang and R. Pan. Convergence rate for compressible Euler equations with damping and vacuum, Arch. Rational Mech. Anal. 166 (2003) 359-376.

[19] F. Huang, P. Marcati and R. Pan. Convergence to Barenblatt solution for the compressible Euler equations with damping and vacuum, Arch. Rational Mech. Anal. 176 (2005) 1-24.

[20] D. Iftimie. The resolution of the Navier-Stokes equations in anisotropic spaces, Revista Matemática Iberoamericana 15 (1999) 1-36.

[21] T. Iguchi and S. Kawashima. On space-time decay properties of solutions to hyperbolicelliptic coupled systems, Hiroshima Math. J. 32 (2002) 229-308.

[22] T. Kato. The Cauchy problem for quasi-linear symmetric hyperbolic systems, Arch. Rational Mech. Anal. 58 (1975) 181-205.

[23] S. Kawashima and W.-A. Yong. Dissipative structure and entropy for hyperbolic systems of balance laws, Arch. Rational Mech. Anal. 174 (2004) 345-364.

[24] S. Kawashima and W.-A. Yong. Decay estimates for hyperbolic balance laws, J. Anal. Appl. 28 (2009) 1-33.

[25] S. Jin and Z. Xin. The relaxation schemes for systems of conservation laws in arbitrary space dimensions. Comm. Pure and Appl. Math. 48 (1995) 235-276.

[26] A. Majda, Compressible Fluid Flow and Conservation laws in Several Space Variables, Springer-Verlag: Berlin/New York, 1984. 
[27] A. Matsumura. An energy method for the equations of motion of compressible viscous and heat-conductive fluids, MRC Technical Summary Report, Univ. of Wisconsin-Masison, \# 2194 (1981).

[28] A. Matsumura and T. Nishida. The initial value problem for the quations of motion of viscous and heat-conductive gases, J. Math. Kyoto Univ. 20 (1980) 67-104.

[29] T. Nishida. Nonlinear hyperbolic equations and relates topics in fluid dynamics, Publ. Math. D'Orsay (1978) 46-53.

[30] T. Ruggeri and D. Serre. Stability of constant equilibrium state for dissipative balance laws system with a convex entropy. Quart. Appl. Math. 62 (2004) 163-179.

[31] Y. Shizuta and S. Kawashima. Systems of equations of hyperbolic-parabolic type with applications to the discrete Boltzmann equation, Hokkaido Math. J. 14 (1985) 249-275.

[32] T. Sideris, B. Thomases and D. H. Wang. Long time behavior of solutions to the 3D compressible Euler with damping, Comm. P. D. E. 28 (2003) 953-978.

[33] H. Triebel, Theory of function spaces, Birkhäuser, 1983.

[34] W. Wang and T. Yang. The pointwise estimates of solutions for Euler equations with damping in multi-dimensions. J Diff. Eqs. 173 (2001) 410-450.

[35] J. Xu. Relaxation-time limit in the isothermal hydrodynamic model for semiconductors, SIAM J. Math. Anal., 40 (2009) 1979-1991.

[36] W.-A. Yong. Entropy and global existence for hyperbolic balance laws, Arch. Rational Mech. Anal. 172 (2004) 247-266.

[37] Y. Zeng. Gas dynamics in thermal nonequilibrium and general hyperbolic systems with relaxation, Arch. Rational Mech. Anal. 150 (2004) 225-279. 